\title{
A Critical Study of the Elastic Properties and Stability of Heusler Compounds: Phase Change and Tetragonal $X_{2} Y Z$ Compounds
}

\author{
Shuchun Wu${ }^{1}$, S. Shahab Naghavi ${ }^{1,2}$, Gerhard H. Fecher ${ }^{1 *}$, Claudia Felser ${ }^{1}$ \\ ${ }^{1}$ Max Planck Institute for Chemical Physics of Solids, Dresden, Germany \\ ${ }^{2}$ Department of Materials Science and Engineering, Northwestern University, Evanston, IL, USA \\ Email: fecher@cpfs.mpg.de
}

How to cite this paper: Wu, S.C., Naghavi, S.S., Fecher, G.H. and Felser, C. (2018) A Critical Study of the Elastic Properties and Stability of Heusler Compounds: Phase Change and Tetragonal $X_{2} Y Z$ Compounds. Journal of Modern Physics, 9, 775-805. https://doi.org/10.4236/jmp.2018.94050

Received: January 26, 2018

Accepted: March 27, 2018

Published: March 30, 2018

Copyright (c) 2018 by authors and Scientific Research Publishing Inc. This work is licensed under the Creative Commons Attribution International License (CC BY 4.0).

http://creativecommons.org/licenses/by/4.0/

\begin{abstract}
In the present work, the elastic constants and derived properties of tetragonal Heusler compounds were calculated using the high accuracy of the full-potential linearized augmented plane wave (FPLAPW) method. To find the criteria required for an accurate calculation, the consequences of increasing the numbers of $k$-points and plane waves on the convergence of the calculated elastic constants were explored. Once accurate elastic constants were calculated, elastic anisotropies, sound velocities, Debye temperatures, malleability, and other measurable physical properties were determined for the studied systems. The elastic properties suggested metallic bonding with intermediate malleability, between brittle and ductile, for the studied Heusler compounds. To address the effect of off-stoichiometry on the mechanical properties, the virtual crystal approximation (VCA) was used to calculate the elastic constants. The results indicated that an extreme correlation exists between the anisotropy ratio and the stoichiometry of the Heusler compounds, especially in the case of $\mathrm{Ni}_{2} \mathrm{MnGa}$. Metastable cubic $\mathrm{Ni}_{2} \mathrm{MnGa}$ exhibits a very high anisotropy $(\approx 28)$ and hypothetical cubic $\mathrm{Rh}_{2} \mathrm{FeSn}$ violates the Born-Huang stability criteria in the $L 2_{1}$ structure. The bulk moduli of the investigated tetragonal compounds do not vary much $(\approx 130 \cdots 190 \mathrm{GPa})$. The averaged values of the other elastic moduli are also rather similar, however, rather large differences are found for the elastic anisotropies of the compounds. These are reflected in very different spatial distributions of Young's moduli when comparing the different compounds. The slowness surfaces of the compounds also differ considerably even though the average sound velocities are in the same order of magnitude $(3.2 \cdots 3.6 \mathrm{~km} / \mathrm{s})$. The results demonstrate the importance of the elastic properties not only for purely tetragonal Heusler compounds but also for phase change materials that exhibit magnetic shape memory or magnetocaloric effects.
\end{abstract}




\section{Keywords}

Elastic Constants, Elastic Stability, Tetragonal Heusler Compounds,

Cubic Instability, Phase Transition

\section{Introduction}

Heusler-type intermetallic compounds $X_{2} Y Z(X, Y=$ transition metals, and $Z=$ main group elements) have become of particular interest due to their fascinating thermal, electrical, magnetic, and transport properties [1]. The Heusler compounds crystallize in a face-centered cubic ( $\mathrm{fcc}$ ) lattice. They are distinguished in two groups: regular or inverse Heusler compounds. Regular Heusler compounds belong to $F m \overline{3} m$ (space group No. 225) symmetry, and inverse Heusler compounds belong to $F \overline{4} 3 m$ (space group No. 216). Both cubic phases may undergo a cubic-tetragonal phase transition, in which the regular Heusler compounds transform from $F m \overline{3} m$ to the tetragonal $14 / \mathrm{mmm}$ (No. 139), and the inverse Heusler compounds transform from $F \overline{4} 3 m$ to tetragonal $I \overline{4} m 2$ (No. 119). Thus, the parent cubic and obtained tetragonally distorted phases obey a supergroup-subgroup relation.

Due to simple features of Heusler compounds, it is critically important to have an instrument for phase prediction. For example, cubic ferromagnetic Heusler compounds follow the Slater-Pauling rule for localized moment systems. Their magnetic moment $m$ depends simply on the valence electron concentration $n_{v}$ with $m=n_{v}-24$. Further, prospective candidates for superconductivity include certain Heusler compounds with 27 electrons that exhibit a saddle point at the $L$ point close to $E_{\mathrm{F}}$ in the band structure according to the van Hove scenario [2] [3]. On the other hand, a high density of states at the Fermi level causes instability and a phase transition to lower symmetry forced by a band Jahn-Teller distortion [4] [5]. This competition is one example that shows the importance of phase prediction in the Heusler compounds. However, both tetragonal and cubic phases have their own importance for industrial as well as fundamental research.

Tetragonally distorted Heusler compounds have attracted interest in the field of spintronics, in particular, for spin-torque applications, owing to their magnetic anisotropy in the perpendicular axes [6] [7] [8] [9]. Phase change Heusler compounds are interesting as magnetocaloric and magnetic shape memory effect materials [10]. Therefore, the theoretical prediction of new materials with suitable designed properties is active research in this field [11].

In fact, processing and designing new materials requires knowledge of physical properties, such as hardness, elastic constants, melting point, and ductility. The calculation of elastic constants is an efficient and fast tool used for elucidating physical properties as well as the mechanical stability and possible phase transitions of crystalline systems. Applied strains, such as shear or elongation, provide not only valuable information about the instability itself but 
also the directional dependence of instabilities in crystals. The directional dependence of instabilities becomes important when not only cubic-tetragonal but also cubic-hexagonal, tetragonal-hexagonal, and lower symmetry phase transitions are relevant, such as those observed in $\mathrm{Mn}_{3} \mathrm{Ga}$ [6] [7]. Unlike mechanical instability, the determination of elastic constants is essential for applications of magnetic shape memory alloys. The elastic constants also provide valuable information on the structural stability, anisotropic character, and chemical bonding of a system [12] [13] [14]. Moreover, other measurable properties can be estimated using the elastic constants, such as the velocity of sound, Debye temperature, melting point, and hardness. This information is an essential requirement for both industrial applications and fundamental research. For example, these properties are essential for studying superconductivity and heavy fermion systems in which a drastic change of elastic constants and related properties have been reported upon the phase transition [15] [16]. The elastic properties are so important that Gilman [17] concluded: "the most important properties of a crystal are its elastic constants".

In the present study, some well-known tetragonal and cubic Heusler compounds are examined and compared with the available experimental and theoretical data [18]-[26]. Starting from the cubic phase, cubic $L 2_{1} \mathrm{Ni}_{2} \mathrm{MnGa}$ and $\mathrm{Rh}_{2} \mathrm{FeSn}$ are considered for detailed studies. For the tetragonally distorted systems, $\mathrm{Ni}_{2} \mathrm{MnGa}$ (in the non-modulated tetragonal $(c / a>1)$ structure), $\mathrm{Mn}_{2} \mathrm{NiGa}, \mathrm{Fe}_{2} \mathrm{MnGa}$, and $\mathrm{Mn}_{2} \mathrm{FeGa}$ Heusler compounds are examined. The intermetallics $\mathrm{Mn}_{2} Y \mathrm{Ga}(Y=\mathrm{Fe}, \mathrm{Ni})$ and $X_{2} \mathrm{MnGa}(X=\mathrm{Fe}, \mathrm{Ni})$ undergo tetragonal magneto-structural transitions that may result in half metallicity and magnetic shape memory or magnetocaloric effects. In the case of $\mathrm{Ni}_{2} \mathrm{MnGa}$, the composition dependence (chemical disorder effect) of the phase transition is studied using the virtual crystal approximation (VCA). Calculating the mechanical and elastic properties of off-stoichiometric compounds in the tetragonal phases elucidates phase transformations. Elastic constants and mechanical properties of some Rh-based Heusler compounds, reported by Suits [27], are calculated. The dependence of the elastic constants and the number of used $k$-points and plane waves (defined in full-potential linearized augmented plane wave (FPLAPW) by $R_{\mathrm{MT}} k_{\max }$, where $R_{\mathrm{MT}}$ is the muffin tin radius and $k_{\max }$ is the largest $k$ vector) are discussed in detail. The importance of using sufficiently large numbers of $k$-points and plane waves for a reliable estimation of the elastic properties is demonstrated.

The present work concentrates on the elastic properties of metastable cubic, tetragonal, and phase change materials that exhibit magnetic shape memory or magnetocaloric effects.

\section{Methodology}

\subsection{Computational Details}

In this section, the basic equations for calculating the elastic constants are 
presented (for more details see Appendix A). The most easily determined quantity is the bulk modulus $B$, which provides the behavior of the crystal volume or lattice parameters under hydrostatic pressure. There are several ways to calculate the bulk modulus from the energy-volume $E_{\text {tot }}(V)$ relation (see References [28] [29] [30]). In the present work, the bulk modulus $B$ is determined by fitting the total energy calculations to the Birch-Murnaghan equation of state [29] [30]. According to this model, the dependence of the energy on the change in the crystal volume $V$ under hydrostatic pressure $p$ is given by

$$
E=E_{0}+\frac{9}{16} \frac{B V_{0}}{14703.6}\left[\left(6-4 v^{2}\right) \eta^{2}+B^{\prime} \eta^{3}\right]
$$

where $B^{\prime}=\mathrm{d} B / \mathrm{d} p$ is the pressure derivative of the bulk modulus and $\eta=v^{2}-1$, with the ratio $v=\left(V_{0} / V\right)^{1 / 3}$ of the actual volume $V$ under pressure to the relaxed volume $V_{0}$ at the lowest total energy $E_{0}$ (see Reference [31]). The related pressure is given by

$$
p=\frac{3}{2} B\left(v^{7}-v^{5}\right)\left[1+\frac{3}{4}\left(B^{\prime}-4\right) \eta\right] .
$$

For tetragonal crystals, the dependence of the bulk modulus $B$ on the elastic stiffness is given by

$$
B=\frac{1}{9}\left(2 c_{11}+2 c_{12}+4 c_{13}+c_{33}\right) .
$$

In the case of cubic crystals, $c_{12}=c_{13}, c_{11}=c_{33}$, and $c_{44}=c_{66}$ (see also Appendix A). Therefore, the equations of the elastic constants for cubic systems are easily obtained from the tetragonal equations.

The remaining elastic properties are determined by applying different types of strain $\left(e_{i}\right)$ to the tetragonal lattice and by applying proper relations between the total energy and the strain components. The energy $E\left(e_{i}\right)$ of the strained lattice is calculated using Hooke's law (see Equation (10)). According to Wallace [31], if the strains $e_{i}$ are small, the change of the energy is given by

$$
\frac{\Delta E}{V_{0}}=\sum \Delta_{i} e_{i}+\frac{1}{2} \sum \sum c_{i, j} e_{i} e_{j} .
$$

Here, $\Delta E=E\left(e_{i}\right)-E_{0}$ with equilibrium energy $E_{0}$ at volume $V_{0}$ without strain. The linear terms vanish at equilibrium or if the strain causes no change in the volume of the crystal. The elastic constants $c_{i, j}$ are obtained from the second-order terms and are calculated from the second derivatives of the energy with respect to the strains:

$$
c_{i, j}=\left.2 \frac{\partial^{2} E}{\partial e_{i} \partial e_{j}}\right|_{V=V_{0}}
$$

The second derivative relation of elastic constants $\left(c_{i j}\right)$ with total energy highlights the importance of an accurate calculation of the total energy. Therefore, the choice of the density functional theory solver in the calculation of 
elastic constants and related properties is significant.

For cubic crystals, three independent elastic constants need at least two different strains to be applied in the calculations, while in tetragonal systems (space group Nos. 89-142), six independent elastic constants need five different strains. The third (for cubic systems) or sixth (for tetragonal systems) condition to solve the linear set of equations are found from the calculated bulk modulus using the equation of state (see Equations (1) and (3)). In fact, there are numerous ways to apply the six different strains and their combinations to the crystal. One condition is that the applied strains $(\delta)$ should result in different $2^{\text {nd }}$ derivatives of the strain energy given by Equation (4) (see Table 1). Another, necessary side condition of Equation (5) is that the volume must be conserved when applying the strain. Therefore, the use of linear strain components $\left(e_{i}=\delta\right.$ in all possible cases and combinations in the strain matrix of Equation (9) of Appendix A) would lead to large uncertainties because they are not always volume conservative, or they make the use of additional derivatives necessary (for example, $\frac{\partial E}{\partial V}, \frac{\partial V}{\partial e_{i}}$, and higher orders).

Table 1 and Figure 1 summarize the applied strains that are used to determine the elastic constants in the present work. The applied strains are the same as those reported by Kart et al. [32]. The isotropic strain (0) is not used directly for the calculation of the elastic constants, as it gives the same information as discussed for the bulk modulus $B$ (see discussion above). The five strain types (Equations (1)-(5) in Table 1) are chosen to be volume conservative. The last strain type does not conserve the volume, but it keeps the same symmetry as the crystal and thus can be calculated from the energy versus $c / a$ relation [32], where $c / a$ is the ratio of the two independent lattice parameters of the tetragonal crystals.

In the present work, six distortions of each type in the range of $-3 \% \leq \delta \leq+3 \%$ were applied to the relaxed structure with $V_{0}$ from the structural optimization using the Birch-Murnaghan equation of state. For tetragonal systems, the energy $E(\delta)$ versus applied strain curves were fitted to a fourth-order polynomial $E(\delta)=E_{0}+a_{2} \delta^{2}+a_{3} \delta^{3}+a_{4} \delta^{4}$.

Table 1. Strain table for calculation of the elastic constants in tetragonal systems. Note that only types (1) to (5) are volume conservative. Only components with $e_{i} \neq 0$ are given. Type (0) corresponds to calculation of the bulk modulus.

\begin{tabular}{|c|c|c|c|c|}
\hline Type & & Strain & & $\Delta E / V_{0}$ \\
\hline$(0)$ & isotropic & $e_{1}=\delta$ & $e_{2}=\delta$ & (see bulk modulus) \\
\hline (1) & monoclinic & $e_{1}=\delta^{2} /\left(1-\delta^{2}\right)$ & $e_{4}=\delta$ & $2 c_{44} \delta^{2}+O\left(\delta^{4}\right)$ \\
\hline (2) & triclinic & $e_{3}=\delta^{2} /\left(1-\delta^{2}\right)$ & $e_{6}=\delta$ & $2 c_{66} \delta^{2}+O\left(\delta^{4}\right)$ \\
\hline (3) & orthorhombic & $e_{1}=\delta$ & $e_{2}=-\delta$ & $\left(c_{11}-c_{12}\right) \delta^{2}+O\left(\delta^{4}\right)$ \\
\hline (4) & orthorhombic & $e_{1}=\delta$ & $e_{2}=\delta^{2} /\left(1-\delta^{2}\right)$ & $\left(c_{11}-2 c_{13}+c_{33}\right) \delta^{2} / 2+O\left(\delta^{4}\right)$ \\
\hline (5) & tetragonal & $e_{1}=\delta$ & $e_{2}=\delta$ & $e_{3}=-\delta(2+\delta) /(1+\delta)^{2}\left(c_{11}+c_{12}+2 c_{33}-4 c_{13}\right) \delta^{2} / 2+O\left(\delta^{4}\right)$ \\
\hline (6) & tetragonal & $e_{3}=\delta$ & & $c_{33} \delta^{2} / 2$ \\
\hline
\end{tabular}


(a)

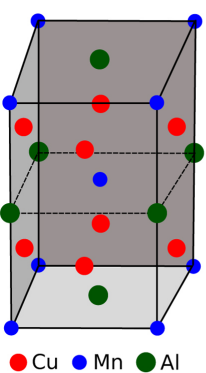

(3)

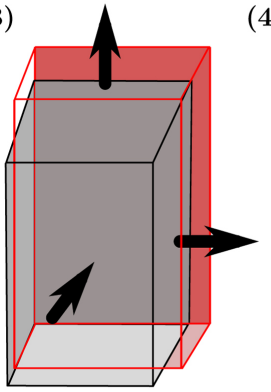

(0)

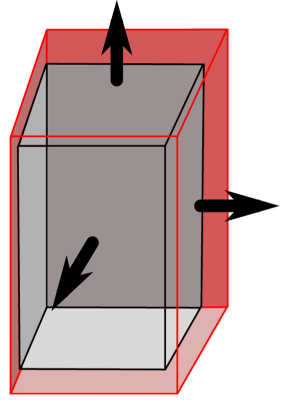

(4)

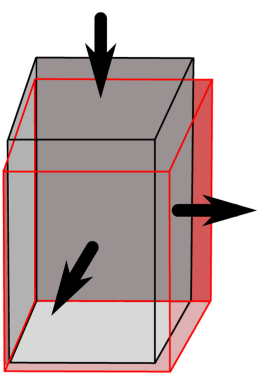

$(1)$

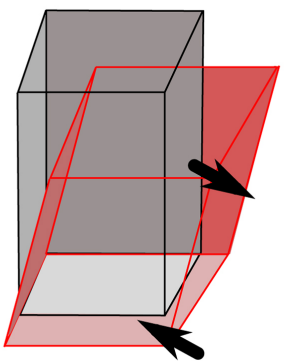

(5)

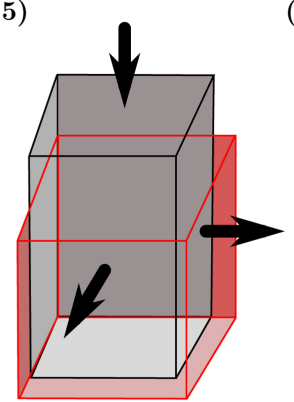

(2)

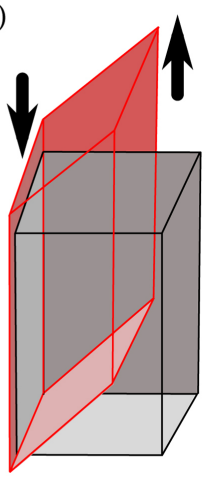

(6)

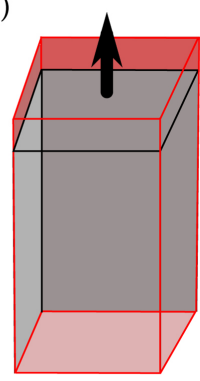

Figure 1. Strain types for calculation of the elastic constants in tetragonal systems. (a) shows the tetragonal Heusler structure with $14 / \mathrm{mmmm}$ symmetry. (0)-(6) show the strain types and resulting distortions according to Table 1.

Here, an alternative method of verifying the convergence as well as the accuracy of the results is introduced. In principle, it is sufficient to use either Equations (4) or (5) of Table 1 to calculate all six elastic constants. This is the usually applied method. However, in the present work the elastic constants and all related properties are calculated with both equations to ensure that they provide the same results. This happens, indeed, only if the results are well converged (see also Section 3.1). The system is overdetermined by using both types of strains; however, in this way, the accuracy of the calculated quantities can be estimated. In fact, the values reported here have an error below $0.5 \%$. The combination of different strains as well as different types of equations of state allow determination of the uncertainty of the calculated results, which is expected for a reliable computer experiment.

\subsection{Electronic Structure Calculations}

The ab-initio electronic structure calculations were performed using the Wien $2 \mathrm{k}$ code [33]. The all-electron full-potential method, FLAPW, with an unbiased basis covers all elements of the periodic table with any spin configuration. This feature is essential for Heusler compounds because they may contain diverse types of atoms, including lanthanide and actinide atoms, together with exotic magnetic ordering. The accuracy of this method makes it suitable for the studied systems. For example, $\mathrm{Co}_{2} \mathrm{TiAl}$ fails with spherical potentials or full symmetry 
potentials together with bare exchange-correlation functionals neglecting gradient corrections [34] [35] [36]. Since elastic constants are calculated from the second derivatives of the total energy, an accurate calculation of total energy is extremely important.

The exchange-correlation functional was taken in the generalized gradient approximation of Perdev, Burke, and Enzerhoff (GGA-PBE) [37] [38]. The number of plane waves was restricted by $R_{\mathrm{MT}} k_{\max }=9$, and the number of $k$-points was set to $8000 k$-points in the full Brillouin zone. As discussed in Section 3.1, these criteria ensure the convergence of the calculated elastic properties for the investigated systems.

The lattice parameters were optimized before calculating the elastic constants. The results of the structural optimizations are summarized in Table 2 along with some previously reported experimental and theoretical values. Here, the $c / a$ ratio was obtained by a full optimization of the Heusler compounds in tetragonal space groups 119 or 139. In other words, to find the energy minimum, not only the $c /$ a ratio changed (the elongation of $c$ ) [32] [39] (see also Figure 2) but also the volume of the structures was relaxed. The assignment of lattice parameters should be performed carefully since the optimization was performed in the tetragonal symmetry. When reducing the cubic $f_{c c}$ cell to a tetragonal $f_{c t}$ cell, the cubic lattice parameter $a=a_{c}$ becomes $c$, and the tetragonal parameter $a=a_{t}$ becomes $a_{c} / \sqrt{2}$. To better understand the distortion of the cubic

Table 2. Results of the structural optimization. Lattice parameters $a$ and $c$ are given in $\AA$, $\mathcal{E}$ is dimensionless, and total magnetic moments $m$ are in $\mu_{\mathrm{B}}$. All structures are fully optimized ( $V, c / a$, magnetic state) for the given symmetries. Column sym gives the number of the corresponding space group, and cubic and tetragonal structures are assigned by the Pearson symbols $c F 16$ and $t I 4$, respectively. Experimental values are given for comparison. Note that the cubic variant of $\mathrm{Rh}_{2} \mathrm{FeSn}$ is only hypothetical.

\begin{tabular}{cccccc}
\hline Compound & sym & $a_{\mathcal{c}} c$ & $a_{t}$ & $\mathcal{E}$ & $m_{\text {tot }}$ \\
\hline $\mathrm{Mn}_{2} \mathrm{NiGa}$ & $t I 4119$ & 6.91 & 3.78 & 0.293 & 1.0 \\
$\mathrm{Ni}_{2} \mathrm{MnGa}$ & $t I 4139$ & 6.80 & 3.78 & 0.272 & 4.0 \\
Exp. [40] & & 6.44 & 3.90 & 0.168 & 4.09 \\
$\mathrm{Ni}_{2} \mathrm{MnGa}$ & $c F 4225$ & 5.81 & & 0 & 4.1 \\
Exp. [41] & & 5.82 & & 0 & 4.17 \\
$\mathrm{Mn}_{2} \mathrm{FeGa}$ & $t I 4119$ & 7.30 & 3.68 & 0.403 & 0.77 \\
$\mathrm{Fe}_{2} \mathrm{MnGa}$ & $t I 4139$ & 7.31 & 3.62 & 0.428 & 0.13 \\
$\mathrm{Rh}_{2} \mathrm{CrSn}$ & $t I 4139$ & 7.33 & 4.08 & 0.270 & 2.40 \\
Exp. [27] & & 7.16 & 4.09 & 0.238 & \\
$\mathrm{Rh}_{2} \mathrm{FeSn}$ & $t I 4139$ & 7.12 & 4.13 & 0.219 & 3.92 \\
Exp. [27] & & 6.91 & 4.15 & 0.177 & 3.70 \\
$\mathrm{Rh}_{2} \mathrm{FeSn}$ & $c F 4225$ & 6.25 & & 0 & 3.54 \\
$\mathrm{Rh}_{2} \mathrm{CoSn}$ & $t I 4139$ & 7.22 & 4.05 & 0.261 & 2.25 \\
Exp. [27] & & 6.90 & 4.14 & 0.179 & 2.44 \\
\hline
\end{tabular}




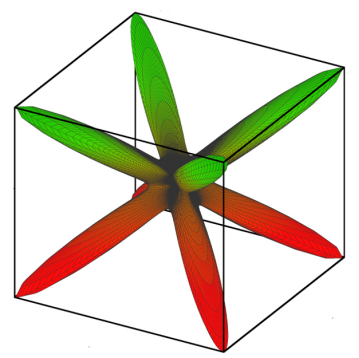

(a)

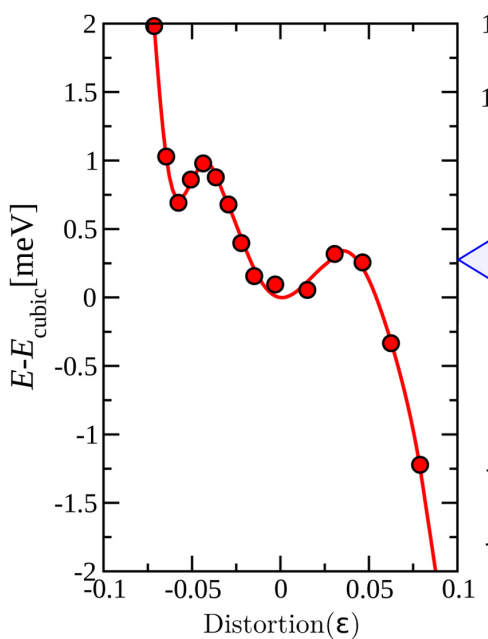

(b)

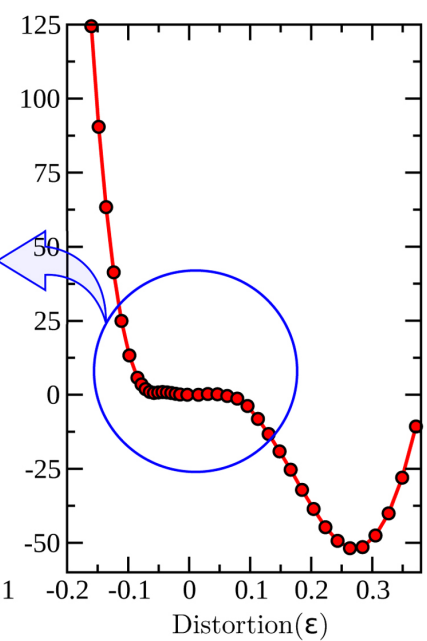

(c)

Figure 2. (a) The calculated spatial distributions of the rigidity modulus $G(\hat{r})$ of $\mathrm{Ni}_{2} \mathrm{MnGa}$. (b, c) The total energy as a function of tetragonal strain along the $c$-axis at constant volume. Panel (b) focuses on the $5 \mathrm{M}$ modulated phase with $c / a \approx 0.9$.

Heusler structure to the tetragonal Heusler structure, the distortion parameter $\mathcal{\varepsilon}$ is defined by

$$
\varepsilon=\frac{c}{a_{t}} \frac{1}{\sqrt{2}}-1,
$$

where $a_{t}$ is the tetragonal a parameter. At $\varepsilon=0$, the structure is cubic; at $\varepsilon<0$, the cubic cell is compressed; and at $\varepsilon>0$, the cubic cell is elongated along one of the principal axes.

The magnetic state was verified using different settings of the initial magnetization: ferromagnetic (all initial spins parallel) or ferrimagnetic (initial spins partially antiparallel). From the studied systems, all $\mathrm{Mn}_{2} \mathrm{YZ}$ compounds exhibit ferrimagnetic order in which one Mn has majority and the other Mn has minority orientation. For all other compounds, the ferromagnetic ground state has the lowest total energy.

\section{Main Results}

Prior to discussing the tetragonal Heusler compounds, the elastic constants of the unstable and metastable cubic systems are discussed. Here, $\mathrm{Ni}_{2} \mathrm{MnGa}$ with 
$L 2_{1}$ structure is selected as a metastable system. This compound is one of the most investigated materials owing to its shape memory behavior and its potential applications in actuator devices. In fact, in Section 3.1, only the cubic phase is addressed, and the tetragonal phase of $\mathrm{Ni}_{2} \mathrm{MnGa}$ is discussed in Section 3.2. Moreover, $\mathrm{Ni}_{2} \mathrm{MnGa}$ was used as an example case to study the significance of increasing the number of $k$-points and plane waves and their relations to the convergence of the elastic constants. In the second part of this section, the tetragonal phase of Heusler compounds are discussed. The elastic constants together with the corresponding measurable properties for selected tetragonal Heusler compounds are investigated. The role of the stoichiometry on the phase transition of $\mathrm{Ni}_{2} \mathrm{MnGa}$ is also explored.

\subsection{Elastic Constants and Metastability in Cubic and Tetragonal Compounds}

Stoichiometric $\mathrm{Ni}_{2} \mathrm{MnGa}$ undergoes a structural phase transition from the austenite into the martensite phase [42]. Depending mostly on the composition, the martensite structure is characterized by the tetragonal $5 M$ modulated structure with $c / a \approx 0.94$, the orthorhombic $7 M$ structure with $c / a \approx 0.9$, and the non-modulated tetragonal structure with $c / a \approx 1.2$ [39]. Figure 2 shows the appearance of different stable and metastable phases with varying $c / a$ elongation. To focus on the cubic phase, Figure 2(b) shows only the small range of strains with $c / a<1$ that cover the cubic phase. The deepest energy minimum is located at a distortion of about $\varepsilon=0.27$, corresponding to $c / a \approx 1.26$ (non-modulated phase). A shallow minimum (see Figure 2(b)) appears at a distortion of about $-0.05(c / a \approx 0.94,5 \mathrm{M}$ phase). The elastic constants of the structure with $c / a>1$ will be discussed in Section 3.2.

As shown in Figure 2(c), the metastable cubic phase exists only under an infinitesimal strain and exhibits a very low energy modulation. The optimized lattice constant of cubic $\mathrm{Ni}_{2} \mathrm{MnGa}$ with the $L 2_{1}$ structure is $5.81 \AA$, in excellent agreement with the experimental value of $5.82 \AA$ [41]. This phase is only stable within $\pm 1 \mathrm{meV}$ energy changes, which confines the lattice distortion to $< \pm 1 \%$. This distortion relates to the tetragonal distortion, providing the $c_{11}-c_{12}$ combination of elastic constants. To observe such a non-trivial change in energy and to have a smooth dependence on strain, the results need to be precisely converged with very high precision. This does not imply, however, that the results do not need to be converged for a wide energy window with a deep minimum. As an example, the importance of converged results is demonstrated in Figure 3. The $c_{44}$ shear modulus is stable for a lattice distortion of about $\pm 3 \%$ and an energy change of more than $\pm 30 \mathrm{meV}$. In this case, the rough calculation provides a smooth curve, but the calculated elastic constants significantly deviate from the converged results. The convergence of the results have the same importance for the calculation of the bulk modulus $(B)$ (see Figure $3(c)$ and Figure 3(d)). 


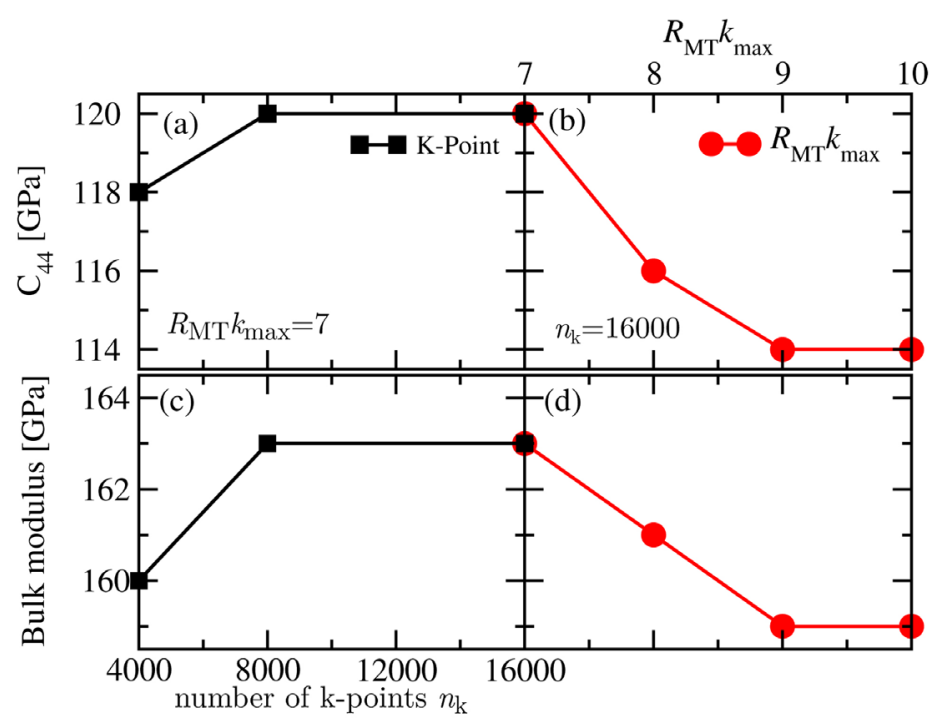

Figure 3. Convergence of elastic properties. The shear modulus $c_{44}$ and bulk modulus $(B)$ as a function of $R_{\mathrm{MT}} k_{\max }$ and the number of $k$-points. The right side panels $(\mathrm{a}, \mathrm{c})$ show the increment of $B$ and $c_{44}$ as a function of the $k$-points with $R_{\mathrm{MT}} k_{\max }=7$. Using the converged $k$-points $(16,000$ or 8000$)$, in the left side panels $(b, d)$, these values decrease with increasing $R_{\mathrm{MT}} k_{\max }$. Therefore, the minimum number of $k$-points and value of $R_{\mathrm{MT}} k_{\max }$ are 8000 and 9 , respectively.

Figure 3 shows the convergence of $c_{44}$ and $B$ with respect to $R_{\mathrm{MT}} k_{\max }$ and number of $k$-points. As shown in $(\mathrm{a}, \mathrm{c})$ for constant $R_{\mathrm{MT}} k_{\max }=7$, increasing the number of $k$-points increases the $c_{44}$ and $B$ values. These results converge at 8000 $k$-points. In contrast, increasing $R_{\mathrm{MT}} k_{\max }$ decreases $c_{44}$ and $B$. Note that a similar result could be obtained at a more relaxed criterion, for example at 2000 $k$-points and $R_{\mathrm{MT}} k_{\max }=7$, due to error cancellations. Therefore, $R_{\mathrm{MT}} k_{\max }=9$ and $8000 \mathrm{k}$-points are the minimum criteria to converge the results for the systems studied in this work.

The calculated elastic constants of $\mathrm{Ni}_{2} \mathrm{MnGa}$ with $L 2_{1}$ structure are given in Table 3. The calculated results show a reasonable agreement with the experimental results and coincide with previously reported theoretical results [32] [39]. Note, however, that good agreement with the experiment results does not guarantee the accuracy of the calculations. First, the experiments were performed at $300 \mathrm{~K}$ for $\mathrm{Ni}_{2} \mathrm{MnGa}$ in the $L 2_{1}$ phase, and off-stoichiometry has a significant effect on the measured elastic constants [44]. Moreover, the employed experimental method may result in different measured elastic constants [43]. As an example, the $c_{44}$ value deviates by about $60 \mathrm{GPa}$ based on the experimental method. In general, the measured elastic constants are inversely related to temperature [45]. Hence, a higher value should be expected for the calculations. Fortunately, $c$, which is a difference between two constants $\left(c_{11}\right.$ and $c_{12}$ ), is argued to be less dependent on temperature [45]. In fact, the calculated $c^{\prime}=4.5 \mathrm{GPa}$ exhibits a better agreement with the experiment 
Table 3. Elastic properties of metastable $\mathrm{Ni}_{2} \mathrm{MnGa}$ and hypothetical $\mathrm{Rh}_{2} \mathrm{FeSn}$ with $L 2_{1}$ structure. The calculated elastic parameters $c_{i p} c^{\prime}$, and $B$ are given in GPa, and the cubic elastic anisotropy $A_{e}=\frac{2 c_{44}}{c_{11}-c_{12}}$ is dimensionless and corresponds to the tetragonal $A_{001}$.

\begin{tabular}{ccccccc}
\hline Compounds & $c_{11}$ & $c_{12}$ & $c_{44}$ & $c^{\prime}$ & $B$ & $A_{e}$ \\
\hline $\mathrm{Ni}_{2} \mathrm{MnGa}$ & 164 & 156 & 115 & 4.1 & 159 & 28.02 \\
Exp. $^{1}$ & 152 & 143 & 103 & 4.5 & 146 & \\
Other calc. $^{2}$ & 163 & 152 & 107 & 5.5 & 156 & \\
$\mathrm{Rh}_{2} \mathrm{FeSn}$ & 124 & 206 & 84.3 & -82 & 179 & $<0$ \\
\hline
\end{tabular}

$(4.1 \mathrm{GPa})$ than previously reported values [32] [39].

In addition, $c_{12}$ and $c_{11}$ do not have any exclusive physical basis; in other words, no phonon mode directly corresponds to these constants. Mixing with other stiffness $\left(c_{i j}\right)$, however, results in a meaningful combination. For example, the tetragonal shear modulus $c^{\prime}=\left(c_{11}-c_{12}\right) / 2$ corresponds to cubic-tetragonal distortion. Moreover, it is well established that $c^{\prime}$-associated with slow transverse acoustic waves [43] - plays an important role in the occurrence of structural transformations. Another important quantity is the Cauchy pressure $c_{p}=c_{12}-c_{44}$. A negative value of $c_{p}\left(c_{12}<c_{14}\right)$ may indicate covalent bonds, where the angular dependence of the inter-atomic forces becomes important.

Furthermore, detailed analysis of elastic constants sheds light on the stability and phase transition in Heusler compounds. Cubic $\mathrm{Ni}_{2} \mathrm{MnGa}$ with soft $c^{\prime}$ is on the border of the phase transition. With the similar interpenetration (see Appendix B), the large elastic anisotropy $A_{e}$ of $\mathrm{Ni}_{2} \mathrm{MnGa}\left(A_{e}=28\right)$ hints on its tendency to deviate from the cubic structure. Anisotropy is another indicator for the instability of cubic structures. The elastic anisotropy of crystals is also an important parameter for engineering since it correlates to the possibility of micro-cracks in materials. Unlike the mechanical properties, anisotropy shows the tendency of a system toward phase transitions as it inversely relates to the $c^{\prime}$ parameter. In fact, an illustrative way to show the anisotropy is to visualize the rigidity modulus $G(\hat{r})$ or Young's modulus $E(\hat{r})$. Figure 2(a) shows that the rigidity modulus is largest in the $\langle 111\rangle$-type direction that is along the tetragonal axes. Such a significant deviation from spherical shape indicates that the moduli of $\mathrm{Ni}_{2} \mathrm{MnGa}$ exhibit a large degree of anisotropy. In principle, when $c_{11}-c_{12} \rightarrow 0$, the rigidity distribution exhibits a stronger directional dependency, as shown for $\mathrm{Ni}_{2} \mathrm{MnGa}$ in Figure 2(a).

In the next step, a compound that is not stable in the cubic structure was examined for comparison. In the case of $\mathrm{Rh}_{2} \mathrm{FeSn}$ shown in Figure 4, the cubic structure exhibits a maximum of the total energy, and any tetragonal distortion will lead to a different stable structure. Based on Figure 4, the appearance of energy minima are expected for two different tetragonally distorted systems with $c / a>1$ and $c / a<1$. Larger distortions show that $c / a>1$ is the stable phase, while $c / a<1$ is a metastable phase. Previous works only reported the structure 


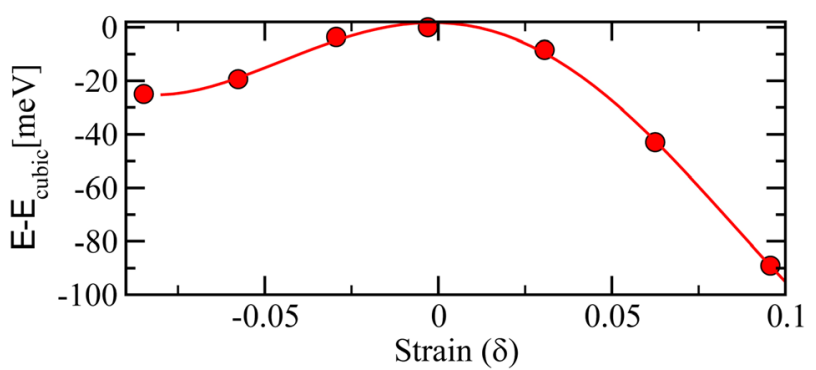

(a)

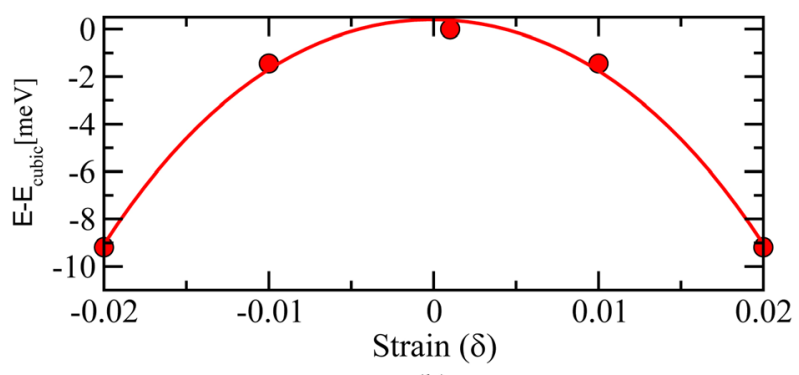

(b)

Figure 4. Energy-strain relation for hypothetical $\mathrm{Rh}_{2} \mathrm{FeSn}$ with $L 2_{1}$ structure. Displayed are the calculated total energies as function of (a) tetragonal (symmetry No. 139) and (b) orthorhombic (symmetry No. 69) strains.

with $\varepsilon>0$ [27] [46]. However, the calculated elastic constants also supported the instability of the cubic phase from negative values of tetragonal shear modulus $c^{\prime}$ and anisotropy $A_{e}$. The metastable structure with $\varepsilon<0$ appears when expanding the in-plane lattice parameter $a$ while keeping $c$ at the cubic lattice parameter. Such a situation may be artificially initialized by epitaxial or pseudomorphic thin film growth on a substrate with an appropriate lattice parameter. Similar metastable situations may exist in many other tetragonal Heusler compounds and will open the field of lattice parameter engineering to enlarge the number of properties on demand.

\subsection{Tetragonal Heusler Compounds}

The tetragonal Heusler compounds studied in this work along with their elastic constants are summarized in Table 4 and Table 5. The Heusler intermetallics $\mathrm{Mn}_{2} Y \mathrm{Ga}(Y=\mathrm{Fe}, \mathrm{Ni})$ and $X_{2} \mathrm{MnGa}(X=\mathrm{Fe}, \mathrm{Ni})$ undergo tetragonal magneto-structural transitions that result in half-metallicity, magnetic shape memory, or magneto-electric effects. In this section, the off-stoichiometric compositions are briefly discussed, and then, the elastic constants and related properties of the Heusler compounds are analyzed. Calculating the elastic properties of the tetragonal phases illuminates the structural transformations, chemical bonding, and mechanical stability of these intermetallic compounds for applications. Likewise, the elastic properties of Rh-based Heusler compounds synthesized by Suits [27] are calculated. Although $\mathrm{Ni}_{2} \mathrm{MnGa}$ has been widely studied experimentally at different phases, there is no experimental measurement 
Table 4. Elastic properties of selected tetragonal Heusler compounds. The different moduli $(B, G, E)$ and elastic constants $C_{i j}$ are given in $\mathrm{GPa}$, the corresponding elastic compliances $s_{i j}$ are in $(\mathrm{TPa})^{-1}$. The anisotropies $\left(A_{\mathrm{B}}, A_{100}, A_{001}\right)$, Phug's ratio $k$, and Poisson's ratio $v$ are dimensionless. The compressibility $\kappa$ is in $\mathrm{GPa}^{-1}$. For $\mathrm{Ni}_{2} \mathrm{MnGa}$, results taken from Reference [39] are given in brackets ().

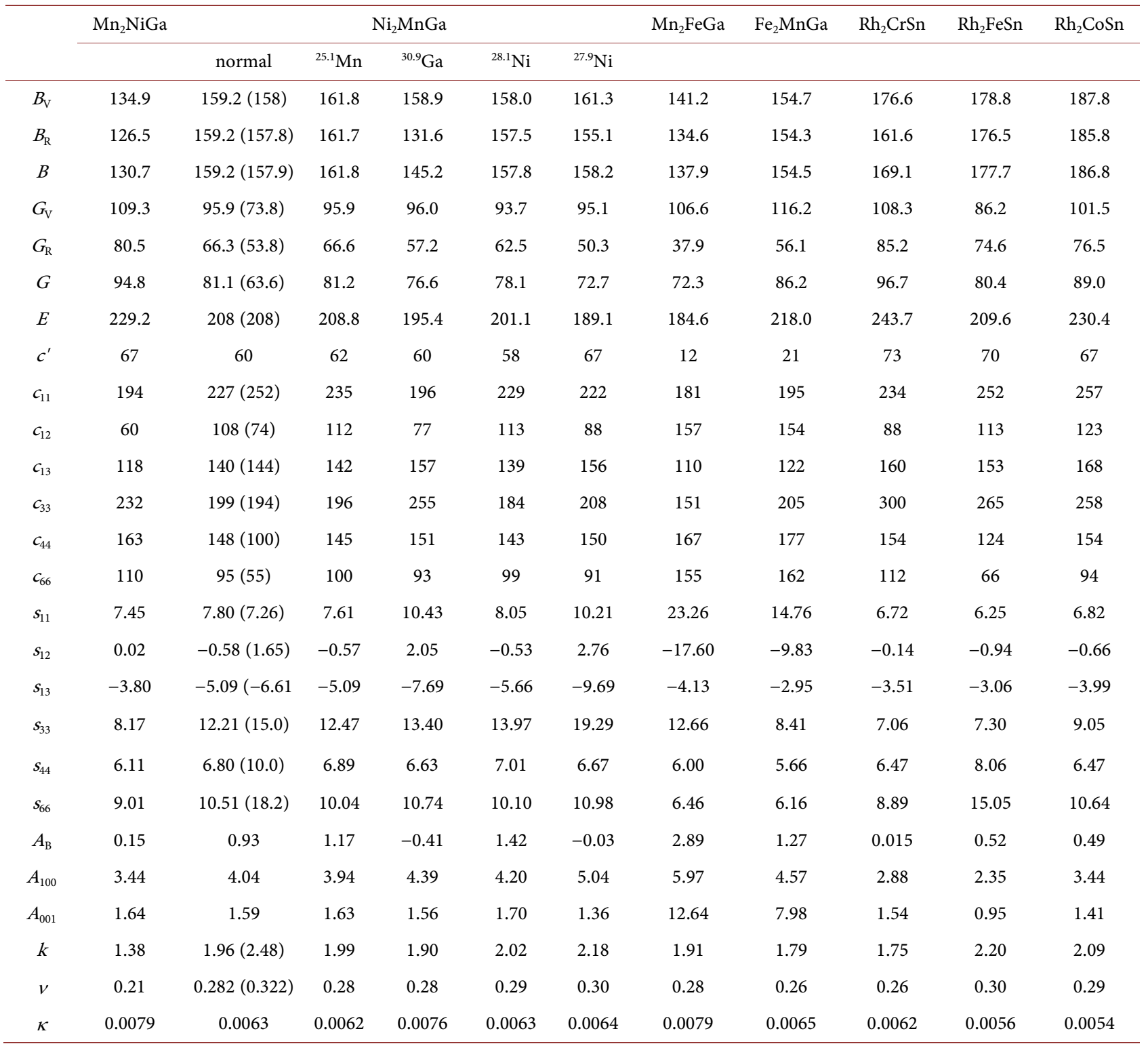

of the elastic modulus of the non-modulated tetragonal phase, and only theoretical works on this phase have already been reported [32] [39].

As shown in Table 4, in the case of $\mathrm{Ni}_{2} \mathrm{MnGa}$, the results show a qualitative agreement with the previous theoretical report (values in brackets). However, a quantitative comparison of the results reveals some significant deviations. These differences can be traced back to the calculation method and the method of performing structural optimization. To address this problem, the cubic phase is briefly considered. In the cubic phase, the present as well as other calculations are performed for the same lattice parameters using different calculation 
Table 5. Derived physical properties of tetragonal Heusler compounds. Tabulated are the longitudinal $v_{l}$, transverse $v_{t}$, and average $\bar{v}$ sound velocities as well as the acoustical Debye temperature $\theta_{\mathrm{D}}^{\mathrm{ac}}$ and acoustical Grühneisen parameter $\zeta^{\mathrm{ac}}$ estimated from the sound velocities. All $v$ are given in $\mathrm{ms}^{-1}, \theta$ is given in $\mathrm{K}$, $\zeta$ is dimensionless.

\begin{tabular}{cccccc}
\hline Compound & $v_{l}$ & $v_{t}$ & $\bar{v}$ & $\theta_{\mathrm{D}}^{\mathrm{ac}}$ & $\zeta^{\mathrm{ac}}$ \\
\hline $\mathrm{Mn}{ }_{2} \mathrm{NiGa}$ & 5667 & 3441 & 3803 & 490 & 1.67 \\
$\mathrm{Ni}_{2} \mathrm{MnGa}$ & 5688 & 3219 & 3487 & 451 & 1.95 \\
Exp. & & & 345 & \\
Other calc. & 5572 & 2853 & 3196 & 323 & \\
$\mathrm{Mn}_{2} \mathrm{FeGa}$ & 5443 & 3023 & 3367 & 434 & 2.02 \\
$\mathrm{Fe}_{2} \mathrm{MnGa}$ & 5753 & 3253 & 3618 & 470 & 1.96 \\
$\mathrm{Rh}_{2} \mathrm{CrSn}$ & 5404 & 3078 & 3421 & 410 & 1.93 \\
$\mathrm{Rh}_{2} \mathrm{FeSn}$ & 5277 & 2803 & 3133 & 376 & 2.19 \\
$\mathrm{Rh}_{2} \mathrm{CoSn}$ & 5346 & 2885 & 3221 & 389 & 2.13 \\
& & & & &
\end{tabular}

schemes. Here, the results of FPLAPW calculations are slightly larger than those of projected augmented wave (PAW) calculations, and the deviations range from $1 \%$ for $c_{11}$ up to $7 \%$ for $c_{44}$. These small differences are expected because of the selected methods and convergence criteria. In contrast, in the case of the tetragonal system (see Table 4), the deviations between calculations range up to $30 \%$, such as the case of $c_{44}$ and $c_{66}$. Indeed, the different calculation methods should not lead to such a large discrepancy (if all factors are set carefully), and these observed differences mainly arise from the underlying structural optimization. Here, all initial tetragonal structures are fully optimized at their relevant symmetries $(I 4 / \mathrm{mmm}$ or $I \overline{4} \mathrm{~m} 2$ ), and thus, they differ from the simply elongated cubic structures (see also Section 2.2 for more details about the calculations).

The elastic constants of all studied tetragonal Heusler compounds follow the inequality $B>c_{44}>G>C^{\prime}>0$, so that the tetragonal shear modulus $c^{\prime}$ is the main constraint on the stability and properties. Pugh's and Poisson's ratio (see Appendix A) supply valuable information about the malleability and the type of bonding in crystals. Small values of Pugh's ratio indicate low malleability of crystals [47], meaning they are brittle. Pugh's ratio indicates the type of bonding, namely covalent or metallic bonding, because changes in the angle of a covalent bond require more energy than stretching-stressing the bond, meaning $G$ becomes larger compared to $B$; this leads to smaller values of $k$. On the other hand, Poisson's ratio for covalent bonding is about $v=0.1$ and increases for ionic crystals up to $v=0.25$. For instance, Pugh's and Poisson's ratios of strong covalent compounds such as diamond are assumed to be $k=0.83$ and $v=0.069$, respectively, while in the strongly ionic KCl, Pugh's and Poisson's ratios are $k=1.19$ and $v=0.27$, respectively [48] [49]. Despite having different types of bonding, both $\mathrm{KCl}$ and diamond are brittle. On the other hand, strongly malleable gold exhibits ratios of $k=6.14$ and $v=0.42$ [47] [50], and 
gold is the most ductile metallic element. According to Christensen [51], the critical value for the ductile-brittle transition appears at a Pugh's ratio of

$$
k_{B / D}=\frac{2}{3} \frac{1}{1-\sqrt{1 / 2}} \approx 2.3,
$$

which corresponds to a critical Poisson's ratio of $v_{B / D}=(3 \sqrt{2}-1)^{-1} \approx 0.31$ (compare equations for $k$ and $v$ in the Appendix). Based on the calculated elastic constants, the studied tetragonal compounds exhibit Pugh's ratios between 1.38 and 2.2 and Poisson's ratios between 0.21 and 0.3 , indicating that they have an intermediate behavior between ductile and brittle type behavior. The values of $k$ and $v$ indicate the covalent or metallic character of these systems. More details about the bonding type may be found from a Bader analysis of the charge densities [52] [53] [54]. It should be kept in mind that ductility and brittleness are rather subjective criteria and usually related to the elastic constants by empirical or semi-empirical rules.

The shear anisotropy factor $A_{\mathrm{e}}$ provides a measure of the degree of anisotropy of the bonds between atoms in different planes. Tetragonal systems are described by two different shear anisotropic factors $A_{001}$ and $A_{100}$ (or equivalent $A_{010}$ ). Young's moduli of some of the studied Heusler compounds are shown in Figure 5. The shear anisotropy for $\{100\}$ planes is considerably higher compared to \{001\} planes for $\mathrm{Mn}_{2} \mathrm{NiGa}$ and $\mathrm{Rh}_{2} \mathrm{FeSn}$. This behavior is similar for $\mathrm{Mn}_{2} \mathrm{FeGa}$ and other $\mathrm{Rh}_{2}$-based compounds, as shown in Table 4. In contrast, in the case of $\mathrm{Fe}_{2} \mathrm{MnGa}$ and $\mathrm{Mn}_{2} \mathrm{FeGa}$, the anisotropy for $\{001\}$ is higher than that for $\{100\}$. Moreover, $\mathrm{Rh}_{2} \mathrm{FeSn}$ has an interesting distribution of Young's modulus: it is isotropic in the square $x-y$ planes of the tetragonal structure, arising from the value of $A_{001}=0.95$, which is close to unity.

\subsection{Virtual Crystal Approximation}

One interesting feature of Heusler compounds, including $\mathrm{Ni}_{2} \mathrm{MnGa}$, is their sensitivity to stoichiometric compositions. An infinitesimal deviation may lead to a phase transition or to changes in the electronic structure properties. Here, VCA was applied to explore off-stoichiometric systems. This approximation is valid for small changes of components with nearly the same radius, which holds for the considered systems. Moreover, VCA is only valid for neighboring elements and for small differences in the number of valence electrons $\left(\Delta e^{-}<0.1\right)$.

In VCA, the atom ${ }^{Z} A$ with charge $Z$ is replaced by atom ${ }^{Z^{\prime}} A$ with the virtual charge $Z^{\prime}=Z \pm \epsilon$ to reflect that the average charge deviates from the original value at a certain position. In $\mathrm{Ni}_{2} \mathrm{MnGa}$, if the site where ${ }^{28} \mathrm{Ni}$ resides is partially occupied by ${ }^{25} \mathrm{Mn}$, the charge at that site will be lower; accordingly, the charge will be higher when ${ }^{30} \mathrm{Ga}$ occupies the same site. In parallel to the change in the nuclear charge, the number of electrons in the primitive cell changes to remain neutral. Thus, for ${ }^{28 \pm \epsilon} \mathrm{Ni}_{2}{ }^{25} \mathrm{Mn}^{31} \mathrm{Ga}$, the number of valence electrons will be $n_{v}=30 \pm 2 \epsilon$ in the primitive cell, whereas the number of core plus semi-core 


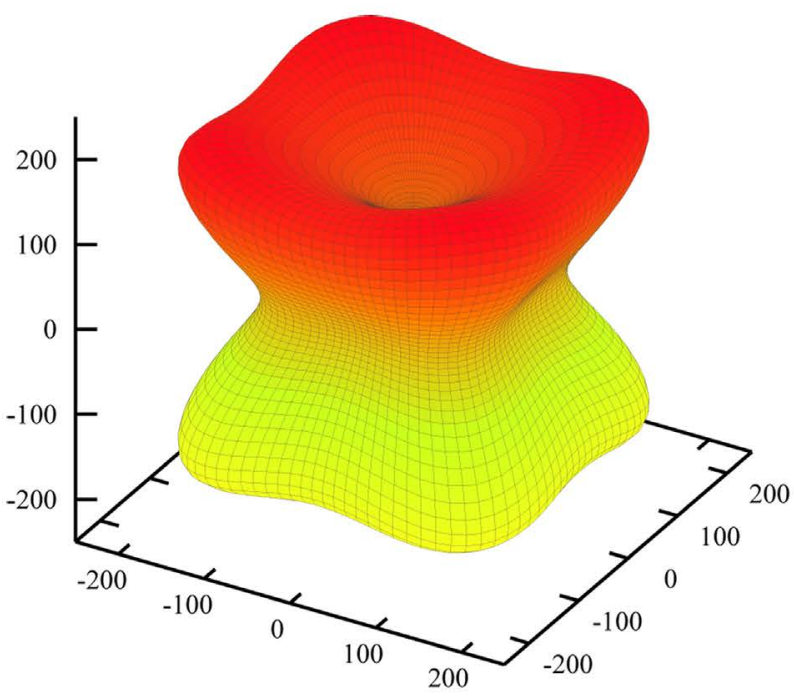

(a)

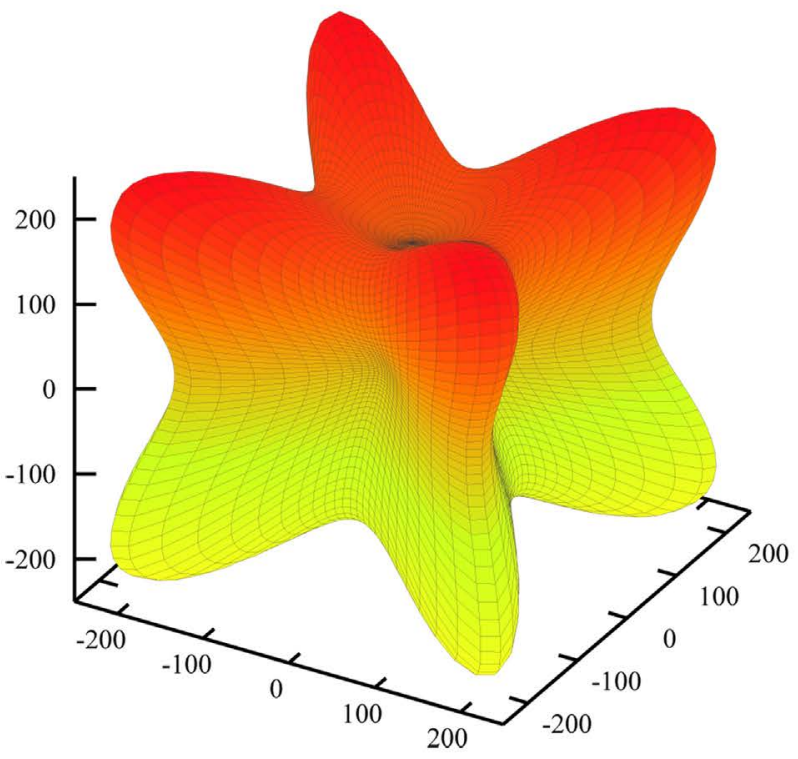

(c)

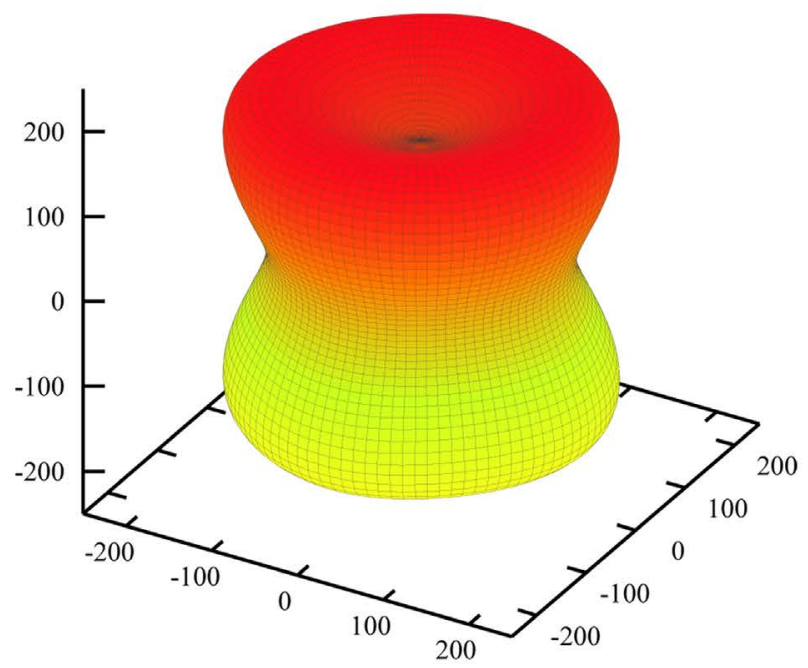

(b)

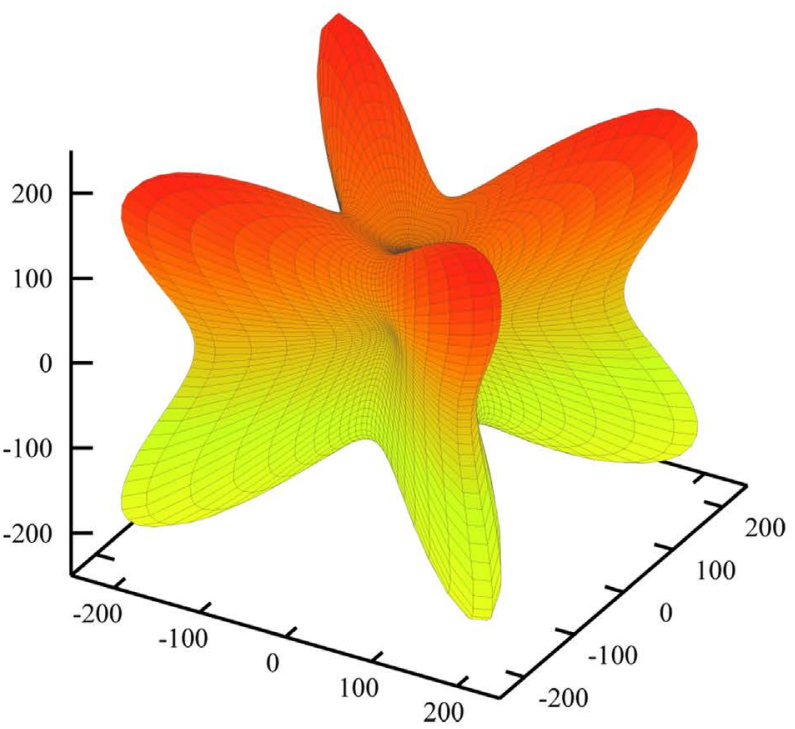

(d)

Figure 5. Calculated spatial distribution of Young's moduli $E(\hat{r})$ of some Mn- and Rh-based Heusler compounds. The change from the round shape of $\mathrm{Rh}_{2} \mathrm{FeSn}$ to the sharply elongated shape in $\mathrm{Mn}_{2} \mathrm{FeGa}$ indicates an increase of the elastic anisotropy (see also Table 4). (a) $\mathrm{Ni}_{2} \mathrm{MnGa}$; (b) $\mathrm{Rh}_{2} \mathrm{FeSn}$; (c) $\mathrm{Fe}_{2} \mathrm{MnGa}$; (d) $\mathrm{Mn}_{2} \mathrm{FeGa}$.

electrons stays fixed at 72. The following cases are used in the present work:

- ${ }^{30.9} \mathrm{Ga} \Rightarrow \mathrm{Ni}$ or $\mathrm{Mn}$ at Ga site,

- ${ }^{25.1} \mathrm{Mn} \Rightarrow \mathrm{Ni}$ or $\mathrm{Ga}$ at $\mathrm{Mn}$ site,

- ${ }^{27.9} \mathrm{Ni} \Rightarrow \mathrm{Mn}$ at $\mathrm{Ni}$ site, and

- ${ }^{28.1} \mathrm{Ni} \Rightarrow \mathrm{Ga}$ at $\mathrm{Ni}$ site.

All elongated structures of off-stoichiometric $\mathrm{Ni}_{2} \mathrm{MnGa}$ have been fully optimized within the VCA approximation. As shown in Figure 6, a small change 


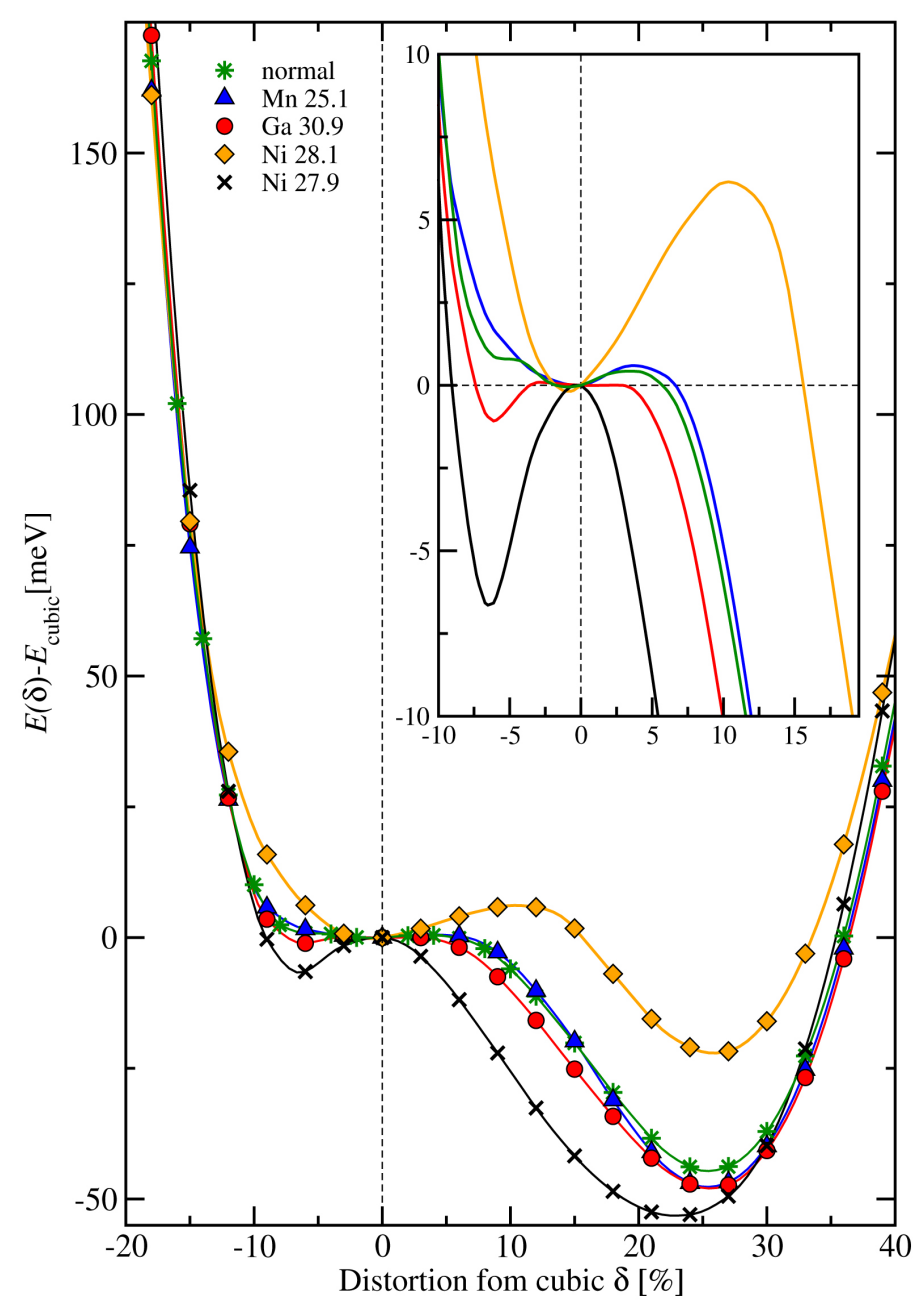

Figure 6. The energy change along $c / a$ is plotted for the non-stoichiometry composition of $\mathrm{Ni}_{2} \mathrm{MnGa}$. The energy landscape (here, along the c-axis) is extremely sensitive to the stoichiometric composition, in particular, the Ni component.

in the number of electrons at the Ni site has the most drastic effect on the energy landscape. The largest difference appears between Ga-rich $\left({ }^{28.1} \mathrm{Ni}\right)$ and Ni-poor $\left({ }^{27.9} \mathrm{Ni}\right)$ compounds. Increasing $\mathrm{Ni}$ at $\mathrm{Ga}$ and $\mathrm{Mn}$ sites lowers the energy minimum at $c / a>1$ compared to the stoichiometric compound. Conversely, increasing $\mathrm{Ga}$ at the Ni site increases the energy of $c / a>1$ with respect to the stoichiometric compound. These results are in agreement with previously reported calculations [55]. Differences in the $E(\delta)$ dependence are more pronounced when the distorted structure is far from the initial structure. In the next step, the elastic constants of the off-stoichiometric $\mathrm{Ni}_{2} \mathrm{MnGa}$ were calculated using VCA for the tetragonal distorted structures with $c / a>1$ at the lowest total energy (see Figure 6).

Table 4 summarizes the results of the elastic constant calculations for stoichiometric and off-stoichiometric $\mathrm{Ni}_{2} \mathrm{MnGa}$. An extreme effect of the off-stoichiometry is reflected in the anisotropy ratio $\left(A_{B}\right)$. As shown in Table 4 , 
the ${ }^{27.9} \mathrm{Ni}$ and ${ }^{30.9} \mathrm{Ga}$ compounds exhibit negative anisotropies of -0.02 and -0.4 , respectively. In fact, the negative anisotropy highlights the extreme instability of these systems. In particular, ${ }^{30.9} \mathrm{Ga}$ has a large negative value. The results explain why ${ }^{30.9} \mathrm{Ga}$ (Ga-poor) and ${ }^{27.9} \mathrm{Ni}$ (Mn-rich) $\mathrm{Ni}_{2} \mathrm{MnGa}$ synthesis is difficult. Moreover, the ${ }^{28.1} \mathrm{Ni}$ (Ga-rich) and ${ }^{25.1} \mathrm{Mn}$ (Mn-poor) compounds have large anisotropies, which are twice the value of the stoichiometric anisotropies. The large value also explains the tendency of phase transitions in these materials. Therefore, off-stoichiometric $\mathrm{Ni}_{2} \mathrm{MnGa}$-nearly all synthesized samples are slightly off-stoichiometric-is expected to have pronounced phase transitions depending on the composition [56]. Thus, deficiency of valence electrons at the $\mathrm{Mn}$ or Ga sites in these systems leads to a negative anisotropy ratio and thus structural instability. Among the elastic constants, $c_{11}$ and $c_{22}$ are more strongly influenced when the composition changes compared to $c_{66}$ and $c_{44}$, which remain nearly constant. Therefore, a small excess of each of the elements (small change of the valence electron concentration in the vicinity of that site) will not change the shear in the $\{100\}$ direction. However, the asymmetries $A_{B}$ and $A_{100}$ significantly change compared to $A_{001}$, reflecting a change of the in-plane chemical bonding. Here, $\mathrm{Ni}_{2} \mathrm{MnGa}$ is used as an example of the sensitivity of Heusler compounds on their stoichiometry. Disorder-induced phase transitions have been reported for other Heusler compounds such as iron-based compounds [57].

\subsection{Derived Properties of Tetragonal Heusler Compounds}

Finally, some physical properties and material parameters of the compounds are derived from the calculated elastic properties. The velocity of sound is an important quantity. Its averaged values can be directly determined from the calculated elastic constants. In experiments, on the other hand, the sound velocities can be used to measure elastic constants. Therefore, the sound velocities $v$ are nearly synonymous with the elastic stiffness constants $c$. Further, sound velocities have been used to study various solid-state properties and processes [45]. Therefore, having the sound velocities predicted by calculations in advance could be quite important for experimental measurements. Usually, the directionally dependent acoustic properties are analyzed in terms of the slowness that is the inverse of the phase velocity. The group velocities are found from the derivatives of the slowness.

Figure 7 compares the slowness surfaces of $\mathrm{Fe}_{2} \mathrm{MnGa}$ and $\mathrm{Ni}_{2} \mathrm{MnGa}$. The slowness surfaces reflect the elastic anisotropy in comparison to Figure 5, which shows the distribution of Young's modulus. Three slowness surfaces appear in both cases, representing different polarizations of the sound wave. The pressure $(p)$ wave is longitudinal polarized. Moreover, $p$ has the highest phase velocity and thus the smallest slowness (Figure $7(a)$ and Figure $7(d)$ ). The remaining two surfaces belong to the fast $\left(s_{1}\right)$ and slow $\left(s_{2}\right)$ shear waves that are transversely polarized. The slowness surfaces of the $p$ waves have a similar shape for both materials, and their maxima are found along the $\{001\}$-type principle axes. The 
$\mathrm{Fe}_{2} \mathrm{MnGa}$ (a) p-wave

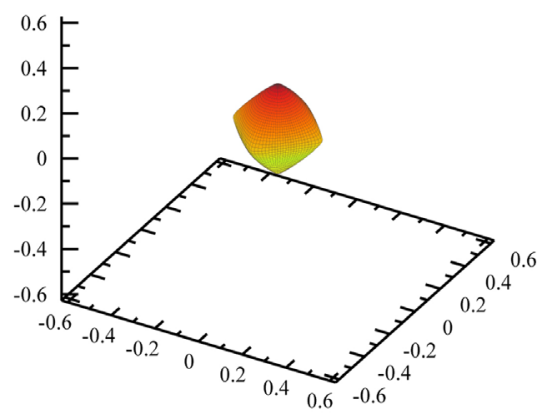

$\mathrm{Ni}_{2} \mathrm{MnGa}$ (d) p-wave

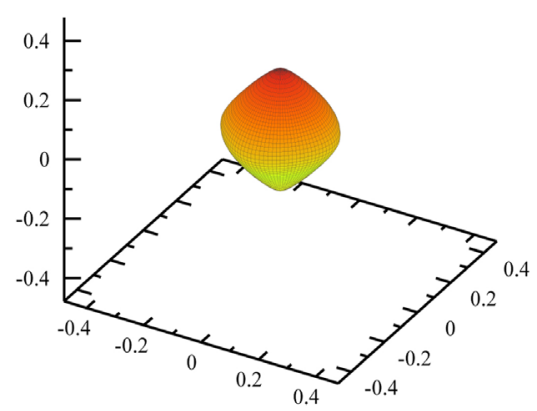

(b) $s_{1}$-wave (fast)

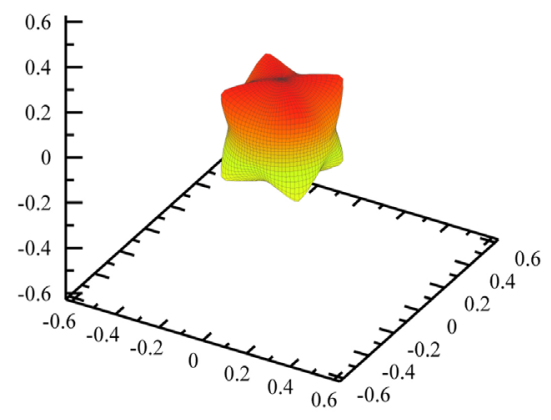

(e) $\mathrm{s}_{1}$-wave (fast)

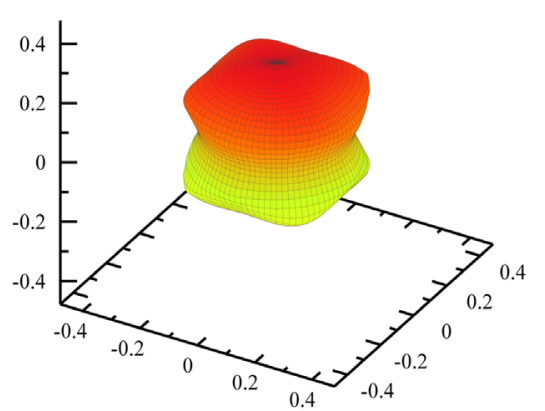

(c) $\mathrm{s}_{2}$-wave (slow)

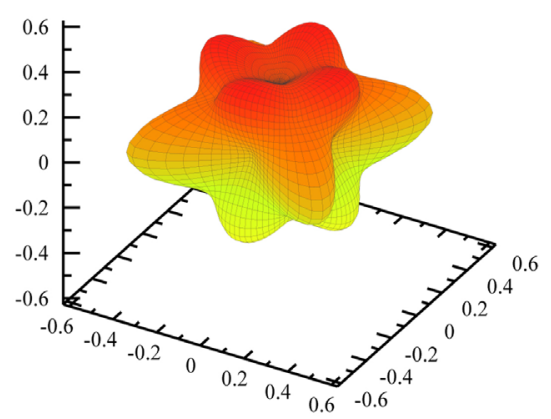

(f) $s_{2}$-wave (slow)

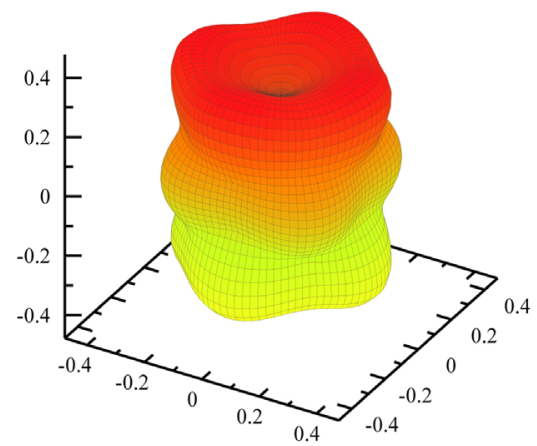

Figure 7. Slowness surfaces of $\mathrm{Fe}_{2} \mathrm{MnGa}$ (a)-(c) and $\mathrm{Ni}_{2} \mathrm{MnGa}(\mathrm{d})-(\mathrm{f})$. The slowness is given in (km/s) ${ }^{-1}$.

shapes of the slowness surfaces of the shear waves differ between the two compounds. The observed differences reflect the differences in the anisotropy of both materials, and it is clearly seen that $\mathrm{Ni}_{2} \mathrm{MnGa}$ has a much lower anisotropy in the $x$ - $y$ plane.

Further material parameters are derived from the average sound velocities as described in Appendix C. At low temperatures, where only acoustic vibrational modes contribute, the Debye temperature $\theta_{\mathrm{D}}$ can be estimated from the average sound velocity [58]. The values estimated in this way are generally larger than Debye temperatures determined from phonon calculations or in experiments [59] because the optical phonon branches are neglected when the elastic constants are used for calculating the "acoustical" Debye temperature $\theta_{\mathrm{D}}^{\text {acc }}$. In a similar way, the average sound velocities can be used to estimate the “acousticaP” Grüneisen parameter $\zeta^{\text {ac }}[60]$.

The calculated average sound velocities together with the estimated Debye temperatures and Grüneisen parameters are listed in Table 5. The average sound velocities are rather similar for all compounds, ranging from about 3100 to 3800 $\mathrm{m} / \mathrm{s}$. The acoustical Debye temperatures are all above room temperature, ranging from 376 to $490 \mathrm{~K}$. As expected, compounds consisting of heavier elements tend to have lower values. With the exception of $\mathrm{Mn}_{2} \mathrm{NiGa}$, all $\zeta^{\text {ac }}$ values are about 2 . This demonstrates that the anharmonicity of the lattice 
vibrations is nearly the same for all compounds.

As further shown in Table 5, the theory and experimental values have about a $20 \%$ discrepancy for the case of $\mathrm{Ni}_{2} \mathrm{MnGa}$. As reported in Reference [61], however, the stoichiometry of the compound has a large effect on the measured Debye temperature. As shown in Table 4, the Debye temperature decreases by about $20 \%$ in ${ }^{27.9} \mathrm{Ni}$. However, in the calculations, the changes in stoichiometry are extremely small. The changes in the calculated values may be more evident with larger variations in the stoichiometry. For example, in experiments, the results change from $261 \mathrm{~K}$ in the case of $\mathrm{Ni}_{49.6} \mathrm{Mn}_{21.9} \mathrm{Ga}_{28.5}$ to $345 \mathrm{~K}$ in the case of $\mathrm{Ni}_{53.1} \mathrm{Mn}_{26.6} \mathrm{Ga}_{20.3}$. Therefore, having an ideal 2:1:1 system, like that assumed in most theories, is not easily possible or may even be impossible from an experimental standpoint. However, the estimation of measurable properties should provide information about the studied system and its potential for applications.

\section{Summary}

In the present work, the elastic constants of tetragonally distorted Heusler compounds were determined. The full-potential LAPW method and the gradient-corrected PBE exchange-correlation functional were employed for all calculations. The relation between the calculated elastic constants and convergence criteria was discussed. Increasing only one of the parameters, such as the $k$-points or $R_{\mathrm{MT}} K_{\max }$, while keeping the other parameter low led to large errors in the calculated elastic constants. Therefore, to calculate both elastic constants accurately, $R_{\mathrm{MT}} K_{\max }$ and $k$-points must be sufficiently large to guarantee convergance. Structural optimization was shown to have an important effect on the elastic constants for tetragonal Heusler compounds. The method was used to investigate the crystalline stability of materials based on the calculation of their elastic properties.

Based on the calculated results, the considered tetragonal Heusler compounds are intermediate materials, between brittle and ductile. Elastically, they exhibit mainly metallic rather than covalent bonding. The structural instability, mechanical properties, structural anisotropy, and other mechanical properties were also explored. Using the virtual crystal approximation, the importance of the stoichiometric composition for $\mathrm{Ni}_{2} \mathrm{MnGa}$ was demonstrated, and extreme sensitivity on the variation of the $\mathrm{Ni}$ component in $\mathrm{Ni}_{2} \mathrm{MnGa}$ was observed. Negative anisotropy of ${ }^{27.9} \mathrm{Ni}_{2} \mathrm{MnGa}$ and $\mathrm{Ni}_{2} \mathrm{Mn}^{30.9} \mathrm{Ga}$ together with the large anisotropy of the ${ }^{28.1} \mathrm{Ni}_{2} \mathrm{MnGa}$ and $\mathrm{Ni}_{2}{ }^{25.1} \mathrm{MnGa}$ compounds indicated instability of off-stoichiometric $\mathrm{Ni}_{2} \mathrm{MnGa}$ in the tetragonal phase.

The calculated material properties are useful for applications focusing on bulk materials. However, the appearance and prediction of metastable tetragonal structures allow lattice parameter engineering with artificial $c / a$ ratios initialized by epitaxial or pseudomorphic thin film growth. Thus, Heusler thin films could be designed to have specific properties. 


\section{References}

[1] Graf, T., Felser, C. and Parkin, S.S.P. (2011) Progress in Solid State Chemistry, 39, 1. https://doi.org/10.1016/j.progsolidstchem.2011.02.001

[2] Winterlik, J., Fecher, G.H., Thomas, A. and Felser, C. (2009) Physical Review B, 79, Article ID: 064508. https://doi.org/10.1103/PhysRevB.79.064508

[3] van Hove, L. (1953) Physical Review, 89, 1189. https://doi.org/10.1103/PhysRev.89.1189

[4] Brown, P.J., Bargawi, A.Y., Crangle, J., Neumann, K.-U. and Ziebeck, K. (1999) Journal of Physics. Condensed Matter, 11, 4715. https://doi.org/10.1088/0953-8984/11/24/312

[5] Blum, C.G.F., Ouardi, S., Fecher, G.H., Balke, B., Kozina, X., Stryganyuk, G., Ueda, S., Kobayashi, K., Felser, C., Wurmehl, S. and Büchner, B. (2011) Applied Physics Letters, 98, Article ID: 252501. https://doi.org/10.1063/1.3600663

[6] Winterlik, J., Balke, B., Fecher, G.H., Felser, C., Alves, M.C.M., Bernardi, F. and Morais, J. (2008) Physical Review B, 77, Article ID: 054406.

https://doi.org/10.1103/PhysRevB.77.054406

[7] Krén, E. and Kádár, G. (1970) Solid State Communications, 8, 1653-1655. https://doi.org/10.1016/0038-1098(70)90484-9

[8] Wu, F., Mizukami, S., Watanabe, D., Naganuma, H., Oogane, M., Ando, Y. and Miyazaki, T. (2009) Applied Physics Letters, 94, Article ID: 122503. https://doi.org/10.1063/1.3108085

[9] Wu, F., Mizukami, S., Watanabe, D., Sajitha, E.P., Naganuma, H. and Oogane, M. (2010) IEEE Transactions on Magnetics, 46, 1863-1865. https://doi.org/10.1109/TMAG.2010.2045108

[10] Zhukov, A. (Ed.) (2016) Novel Functional Magnetic Materials: Fundamentals and Applications. In: Springer Series in Material Science, Volume 231, Springer Verlag, Berlin, Heidelberg, New York.

[11] Gilleßen, M. and Dronskowski, R. (2010) Journal of Computational Chemistry, 31, 612.

[12] Gilman, J.J. (2009) Chemistry and Physics of Mechanical Hardness. Johns Wiley and Sons, Inc., Hoboken, New Jersey. https://doi.org/10.1002/9780470446836

[13] Gilman, J.J. (2001) Electronic Basis of the Strength of Materials. Cambridge University Press, Cambridge. https://doi.org/10.1017/CBO9780511541247

[14] Sen, K.D. (Ed.) (1997) Chemical Hardness. In: Structure and Bonding, Volume 80, Springer Verlag, Berlin Heidelberg New York.

[15] Bruls, G., Wolf, B., Finsterbusch, D., Thalmeier, P., Kouroudis, I., Sun, W., Assmus, W., Lüthi, B., Lang, M., Gloos, K., Steglich, F. and Modler, R. (1994) Physical Review Letters, 72, 1754. https://doi.org/10.1103/PhysRevLett.72.1754

[16] Ledbetter, H.M., Kim, S.A., Goldfarb, R.B. and Togano, K. (1989) Physical Review $B, 39,9689$. https://doi.org/10.1103/PhysRevB.39.9689

[17] Gilman, J.J. (1960) Australian Journal of Physics, 13, 327-346. https://doi.org/10.1071/PH600327a

[18] Li, J., Zhang, Z.D., Sun, Y.B., Zhang, J., Zhou, G.X., Luo, H.Z. and Liu, G.D. (2013) Physica B: Condensed Matter, 409, 35-41. https://doi.org/10.1016/j.physb.2012.10.006

[19] Li, C.-M., Luo, H.-B., Hu, Q.-M., Yang, R., Johansson, B. and Vitos, L. (2012) Physical Review B, 86, Article ID: 214205. https://doi.org/10.1103/PhysRevB.86.214205 
[20] Luo, H.-B., Hu, Q.-M., Li, C.-M., Yang, R., Johansson, B. and Vitos, L. (2012) Physical Review B, 86, Article ID: 024427. https://doi.org/10.1103/PhysRevB.86.024427

[21] Li, C.-M., Luo, H.-B., Hu, Q.-M., Yang, R., Johansson, B. and Vitos, L. (2011) Physical Review B, 84, Article ID: 174117. https://doi.org/10.1103/PhysRevB.84.174117

[22] Li, C.-M., Luo, H.-B., Hu, Q.-M., Yang, R., Johansson, B. and Vitos, L. (2011) Physical Review B, 84, Article ID: 024206. https://doi.org/10.1103/PhysRevB.84.024206

[23] Hu, Q.-M., Li, C.-M., Yang, R., Kulkova, S.E., Bazhanov, D.I., Johansson, B. and Vitos, L. (2009) Physical Review B, 79, Article ID: 144112. https://doi.org/10.1103/PhysRevB.79.144112

[24] Moya, X., Manosa, L., Planes, A., Krenke, Th., Acet, M., Morin, M., Zarestky, J.L. and Lograsso, T.A. (2006) Physical Review B, 74, Article ID: 024109. https://doi.org/10.1103/PhysRevB.74.024109

[25] Bungaro, C., Rabe, K.M. and DalCorso, A. (2003) Physical Review B, 68, Article ID: 134104. https://doi.org/10.1103/PhysRevB.68.134104

[26] de Jong, M., Olmsted, D.L., van de Walle, A. and Asta, M. (2012) Physical Review B, 86, Article ID: 224101. https://doi.org/10.1103/PhysRevB.86.224101

[27] Suits, J.C. (1976) Solid State Communications, 18, 423-425. https://doi.org/10.1016/0038-1098(76)90040-5

[28] Ziambaras, E. and Schröder, E. (2003) Physical Review B, 68, Article ID: 064112. https://doi.org/10.1103/PhysRevB.68.064112

[29] Birch, F. (1947) Physical Review, 71, 809. https://doi.org/10.1103/PhysRev.71.809

[30] Murnaghan, F.D. (1944) Proceedings of the National Academy of Sciences of the United States of America, 30, 244. https://doi.org/10.1073/pnas.30.9.244

[31] Wallace, D.C. (1972) Thermodynamics of Crystals. Dover Publication Inc., Mineola, New York.

[32] Özdemir Kart, S., Uludgân, M., Karaman, I. and Câgin, T. (2008) Physica Status Solidi (a), 205, 1026. https://doi.org/10.1002/pssa.200776453

[33] Blaha, P., Schwarz, K., Madsen, G.K.H., Kvasnicka, D. and Luitz, J. (2001) WIEN2k, an Augmented Plane Wave + Local Orbitals Program for Calculating Crystal Properties. Karlheinz Schwarz, Techn. Universität Wien, Austria.

[34] Graf, T., Fecher, G.H., Barth, J., Winterlik, J. and Felser, C. (2009) Journal of Physics D: Applied Physics, 42, Article ID: 084003. https://doi.org/10.1088/0022-3727/42/8/084003

[35] Ishida, S., Akazawa, S., Kubo, Y. and Ishida, J. (1982) Journal of Physics F: Metal Physics, 12, 1111. https://doi.org/10.1088/0305-4608/12/6/012

[36] Mohn, P., Blaha, P. and Schwarz, K. (1995) Journal of Magnetism and Magnetic Materials, 140-144, 183-184. https://doi.org/10.1016/0304-8853(94)00941-4

[37] Perdew, J.P., Burke, K. and Ernzerhof, M. (1997) Physical Review Letters, 78, 1396. https://doi.org/10.1103/PhysRevLett.78.1396

[38] Perdew, J.P., Burke, K. and Ernzerhof, M. (1996) Physical Review Letters, 77, 3865. https://doi.org/10.1103/PhysRevLett.77.3865

[39] Özdemir Kart, S. and Câgin, T. (2010) Journal of Alloys and Compounds, 508, 177-183. https://doi.org/10.1016/j.jallcom.2010.08.039

[40] Martynov, V.V. and Kokorin, V.V. (1992) Journal de Physique III, 2, 739-749. https://doi.org/10.1051/jp3:1992155

[41] Webster, P.J., Ziebeck, K.R.A., Town, S.L. and Peak, M.S. (1984) Philosophical 
Magazine B, 49, 295-310. https://doi.org/10.1080/13642817408246515

[42] Tsunegi, S., Sakuraba, Y., Oogane, M., Takanashi, K. and Ando, Y. (2008) Applied Physics Letters, 93, Article ID: 112506. https://doi.org/10.1063/1.2987516

[43] Worgull, J., Petti, E. and Trivisonno, J. (1996) Physical Review B, 54, Article ID: 15695. https://doi.org/10.1103/PhysRevB.54.15695

[44] Hu, Q.-M., Li, C.-M., Yang, R., Kulkova, S.E., Bazhanov, D.I., Johansson, B. and Vitos, L. (2009) Physical Review B, 79, Article ID: 144112.

https://doi.org/10.1103/PhysRevB.79.144112

[45] Ledbetter, H. (2006) Materials Science and Engineering: A, 442, 31-34. https://doi.org/10.1016/j.msea.2006.04.147

[46] Dhar, S.K., Grover, A.K., Malik, S.K. and Vijayaraghavan, R. (1980) Solid State Communications, 33, 545-547. https://doi.org/10.1016/0038-1098(80)90856-X

[47] Pugh, S.F. (1954) Philosophical Magazine, 45, 823-843. https://doi.org/10.1080/14786440808520496

[48] McSkimin, H.J. and Bond, W.L. (1957) Physical Review, 105, 116. https://doi.org/10.1103/PhysRev.105.116

[49] Lazarus, D. (1949) Physical Review, 76, 545. https://doi.org/10.1103/PhysRev.76.545

[50] Niu, H.Y., Chen, X.-Q., Liu, P.T., Xing, W.W., Cheng, X.Y., Li, D.Z. and Li, Y.Y. (2012) Scientific Reports, 2, Article Number: 718. https://doi.org/10.1038/srep00718

[51] Christensen, R.M. (2013) The Theory of Materials Failure. Oxford University Press, Oxford. https://doi.org/10.1093/acprof:oso/9780199662111.001.0001

[52] Bader, R.F.W. (1990) Atoms in Molecules. A Quantum Theory. Oxford University Press, Oxford.

[53] Otero-de-la Roza, A., Blanco, M.A., Pendas, A.M. and Luana, V. (2009) Computer Physics Communications, 180, 157-166. https://doi.org/10.1016/j.cpc.2008.07.018

[54] Otero-de-la Roza, A., Johnson, E.R. and Luana, V. (2014) Computer Physics Communications, 185, 1007-1018. https://doi.org/10.1016/j.cpc.2013.10.026

[55] Zayak, A.T. and Entel, P. (2004) Materials Science and Engineering. A, 378, 419-423. https://doi.org/10.1016/j.msea.2003.10.368

[56] Jiang, C., Gong, S. and Xu, H. (2003) Materials Science and Engineering: A, 342, 231-235. https://doi.org/10.1016/S0921-5093(02)00288-5

[57] Gasi, T., Ksenofontov, V., Kiss, J., Chadov, S., Nayak, A.K., Nicklas, M., Winterlik, J., Schwall, M., Klaer, P., Adler, P. and Felser, C. (2013) Physical Review B, 87, Article ID: 064411. https://doi.org/10.1103/PhysRevB.87.064411

[58] Anderson, O.L. (1963) Journal of Physics and Chemistry of Solids, 24, 909-917. https://doi.org/10.1016/0022-3697(63)90067-2

[59] Ouardi, S., Fecher, G.H., Felser, C., Schwall, M., Naghavi, S.S., Gloskovskii, A., Balke, B., Hamrle, J., Postava, K., Pistora, J., Ueda, S. and Kobayashi, K. (2012) Physical Review B, 86, Article ID: 045116. https://doi.org/10.1103/PhysRevB.86.045116

[60] Belomestnykh, V.N. (2004) Technical Physics Letters, 30, 91-93. https://doi.org/10.1134/1.1666949

[61] Chernenko, V.A., Fujita, A., Besseghini, S. and Pérez-Landazabal. J.I. (2008) Journal of Magnetism and Magnetic Materials, 320, e156-159. https://doi.org/10.1016/j.jmmm.2008.02.139

[62] Nye, J.F. (1985) Physical Properties of Crystals. Oxford Science Publications, Ox- 
ford.

[63] Voigt, W. (1928) Lehrbuch der Kristallphysik. Teubner Verlag, Leipzig.

[64] Reuß, A. (1929) Berechnung der Fließgrenze von Mischkristallen auf Grund der Plastizit" atitsbedingung f"ur Einkristalle. Ztschr. f. angew. Math. und Meceitschrift für Angewandte Mathematik und Mechanikh., 9, 49.

[65] Hill, R. (1952) Proceedings of the Physical Society. Section A, A65, 349. https://doi.org/10.1088/0370-1298/65/5/307

[66] Born, M. (1940) Mathematical Proceedings of the Cambridge Philosophical Society, 36, 160-172. https://doi.org/10.1017/S0305004100017138

[67] Misra, R.D. (1940) Mathematical Proceedings of the Cambridge Philosophical Society, 36, 173-182. https://doi.org/10.1017/S030500410001714X

[68] Born, M. and Fürth, R. (1940) Mathematical Proceedings of the Cambridge Philosophical Society, 36, 454-465. https://doi.org/10.1017/S0305004100017503

[69] Born, M. and Misra, R.D. (1940) Mathematical Proceedings of the Cambridge Philosophical Society, 36, 466-478. https://doi.org/10.1017/S0305004100017515

[70] Fürth, R. (1941) Mathematical Proceedings of the Cambridge Philosophical Society, 37, 34-54. https://doi.org/10.1017/S0305004100021514

[71] Fürth, R. (1941) Mathematical Proceedings of the Cambridge Philosophical Society, 37, 177-185. https://doi.org/10.1017/S0305004100021666

[72] Born, M. and Huang, K. (1956) Dynamical Theory of Crystal Lattices. Clarendon Press, Oxford. 


\section{Appendix}

\section{Appendix A. Basic Equations for the Elastic Constants, Moduli, and Related Parameters}

The equations that describe the elastic properties of solids have been described in detail by Nye [62]; this discussion is summarized here and compares tetragonal, hexagonal, and cubic systems with focus on the tetragonal case. The strain matrix $\epsilon$ transforms lattice $A$ with basis vectors $X, Y, Z$ into the deformed lattice

$$
A^{\prime}=(\underline{1}+\epsilon) A
$$

with basis vectors $X^{\prime}, Y^{\prime}, Z^{\prime}$. The symmetric strain matrix $\epsilon$ contains six different strains $e_{i}$ and has the form (numbering of the elements follows Reference [62]):

$$
1+\epsilon=\left(\begin{array}{ccc}
1+e_{1} & \frac{1}{2} e_{6} & \frac{1}{2} e_{5} \\
\frac{1}{2} e_{6} & 1+e_{2} & \frac{1}{2} e_{4} \\
\frac{1}{2} e_{5} & \frac{1}{2} e_{4} & 1+e_{3}
\end{array}\right)
$$

The elastic relations (Hooke's law) between the strain $(\epsilon)$ and stress $(\sigma)$ matrices are mediated by the elastic compliance $(S)$ or the elastic stiffness $(C)$ matrices:

$$
\begin{gathered}
\epsilon=\boldsymbol{S} \sigma \\
\text { or } \sigma=\boldsymbol{C} \epsilon
\end{gathered}
$$

From the elastic equations, the relations between the compliance matrix and the stiffness matrix are

$$
\boldsymbol{S}=\boldsymbol{C}^{-1}
$$

and vice versa $\boldsymbol{C}=\boldsymbol{S}^{-1}$. These relations imply that $\boldsymbol{S C}=\boldsymbol{C S}=\underline{1}$.

In the most general case, the elastic matrix is symmetric and of order $6 \times 6$. In triclinic lattices, the elastic matrix contains 21 independent elastic constants. This number is largely reduced in high symmetry lattices. For example, in an isotropic system, it contains only the two constants $c_{11}=c_{22}=c_{33}$ and $c_{12}=c_{13}=c_{23}$, and the remaining diagonal elements of the matrix are determined by $c_{44}=c_{55}=c_{66}=\left(c_{11}-c_{12}\right) / 2$.

In cubic lattices, the three elastic constants $c_{11}, c_{12}$, and $c_{44}$ are independent. There are five independent elastic constants for hexagonal structures $\left(c_{11}, c_{12}, c_{13}, c_{33}\right.$, and $\left.c_{44}\right)$, while tetragonal structures have either seven (classes: $4, \overline{4}$, or $4 / m)$ or six $\left(c_{11}, c_{12}, c_{13},\left(c_{16}=-c_{15}\right), c_{33}, c_{44}\right.$ and $c_{66}$ ) elastic constants. The elastic matrix for all classes of cubic and hexagonal crystals as well as the classes $4 \mathrm{~mm}, \overline{4} 2 \mathrm{~m}, 422$, or $4 / \mathrm{mmm}$ of tetragonal crystals have the form 


$$
C=\left(\begin{array}{cccccc}
c_{11} & c_{12} & c_{13} & . & . & c_{16} \\
c_{12} & c_{11} & c_{13} & \cdot & . & -c_{16} \\
c_{13} & c_{13} & c_{33} & \cdot & . & \cdot \\
\cdot & \cdot & \cdot & c_{44} & \cdot & \cdot \\
\cdot & \cdot & \cdot & \cdot & c_{44} & \cdot \\
c_{16} & -c_{16} & \cdot & . & \cdot & c_{66}
\end{array}\right)
$$

where zero elements are assigned by dots and additional tetragonal elements for classes $4, \overline{4}$, or $4 / m$ are given in brackets. Moreover, the elastic matrix has restrictions $c_{33}=c_{11}, c_{66}=c_{44}, c_{13}=c_{12}$ in cubic systems and $c_{66}=\left(c_{11}-c_{12}\right) / 2$ in hexagonal systems.

The matrix $C^{\text {tetra }}$ has six eigenvalues for the classes $4 m m, \overline{4} 2 m, 422$, or 4/mmm:

- $C_{1}^{t}=c_{11}-c_{12}$,

- $C_{2,3}^{t}=\frac{1}{2}\left(c_{11}+c_{33}+c_{12} \pm \sqrt{Z}\right)$

- $C_{4}^{t}=c_{66}$, and

- $C_{5,6}^{t}=C_{44}$,

where $Z=c_{11}^{2}+2 c_{11} c_{12}-2 c_{11} c_{33}+c_{12}^{2}-2 c_{12} c_{33}+8 c_{13}^{2}+c_{33}^{2}$. The last eigenvalue $\left(C_{5,6}^{t}\right)$ is twofold degenerate (also note the double sign $( \pm$ ) in the second line). The crystal becomes unstable when one of the eigenvalues becomes zero or negative or in case that $Z<0$.

The relations between the elastic constants $c_{i j}$ and the elements of the compliance matrix $s_{i j}$ are found from Equation (11). In all classes of hexagonal systems or in tetragonal systems belonging to the classes $4 \mathrm{~mm}, \overline{4} 2 \mathrm{~m}, 422$, or $4 / \mathrm{mmm}$, the relations between $c_{i j}$ and $s_{i j}$ are given by

$$
\begin{gathered}
s_{11}=\frac{c_{11} c_{33}-c_{13}^{2}}{c\left(c_{11}-c_{12}\right)} \\
s_{12}=\frac{-c_{12} c_{33}+c_{13}^{2}}{c\left(c_{11}-c_{12}\right)} \\
s_{13}=\frac{-c_{13}}{c} \\
S_{33}=\frac{c_{11}+c_{12}}{c} \\
c=c_{33}\left(c_{11}+c_{12}\right)-2 c_{13}^{2} \\
S_{44}=\frac{1}{c_{44}} \\
S_{66}=\frac{1}{c_{66}}
\end{gathered}
$$

where $c_{66}$ appears only in tetragonal systems. Indeed, the number of equations is much less in cubic systems as shown from the restrictions given above.

The elastic properties of single crystals are completely determined by the 
elastic matrices $C$ and $S$. In reality, polycrystalline materials are considered more often than single crystals. Polycrystalline materials consist of randomly oriented crystals, and thus, a description of their elastic properties requires only two independent elastic moduli: the bulk modulus $(B)$ and the shear modulus $(G)$. The relationships between the single-crystal elastic constants and the polycrystalline elastic moduli are given by the Voigt [62] or Reuß [64] averages. Voigt's approach uses the elastic stiffnesses $c_{i j}$, while Reuß's approach uses the compliances $s_{i j}$. Voigt's moduli [63] are given as function of the elastic constants by the equations:

$$
\begin{aligned}
& B_{V}=\frac{1}{9}\left(2 c_{11}+2 c_{12}+4 c_{13}+c_{33}\right) \\
& G_{V}=\frac{1}{15}\left(2 c_{11}-c_{12}-4 c_{13}+c_{33}+6 c_{44}+3 c_{66}\right)
\end{aligned}
$$

and Reuß's moduli [64] are usually calculated from the elements of the compliance matrix:

$$
\begin{aligned}
B_{R} & =\frac{1}{2 s_{11}+2 s_{12}+4 s_{13}+s_{33}}=\frac{\left(c_{11}+c_{12}\right) c_{33}-2 c_{13}^{2}}{c_{11}+c_{12}+2 c_{33}-4 c_{13}} \\
G_{R} & =\frac{15}{8 s_{11}-4 s_{12}-8 s_{13}+4 s_{33}+6 s_{44}+3 s_{66}}
\end{aligned}
$$

For cubic or isotropic crystals, the bulk moduli in Voigt's $\left(B_{V}\right)$ and Reuß's $\left(B_{R}\right)$ approach are equal, as shown by using the restrictions on $c_{i j}$ given above. In cases other than isotropic or cubic, $G_{R}$ cannot be easily rewritten in terms of the elastic constants.

Finally, the mechanical properties of polycrystalline materials are approximated in the Voigt-Reuß-Hill [65] approach, where the bulk and shear moduli are given by arithmetic averages:

$$
\begin{aligned}
& B=\frac{1}{2}\left(B_{V}+B_{R}\right) \\
& G=\frac{1}{2}\left(G_{V}+G_{R}\right)
\end{aligned}
$$

The bulk modulus $B$ of a material characterizes its resistance to fracture, whereas the shear modulus $G$ characterizes its resistance to plastic deformations. Therefore, ratios between the elastic moduli $B$ and $G$ are often given for characterization and comparison of different materials. Pugh's modulus $k$ is the simple ratio of the bulk and shear moduli [47]:

$$
k=B / G \text {. }
$$

Poisson's ratio $v$ also relates the bulk and shear moduli:

$$
v=\frac{1}{2} \frac{3 B-2 G}{3 B+G}=\frac{3 k-2}{6 k+2} .
$$

Further, Poisson's ratio bridges between the rigidity modulus $G$ and Young's modulus $E$, which is given by 


$$
E=2 G(1+v)=\frac{9 B G}{3 B+G}
$$

\section{Appendix B. Elastic Stability and Representation of Elastic Properties}

The set of elastic moduli and their ratios allows characterization of the elastic behavior of materials. However, the mechanical stability is still open. As a first criterion, the elastic moduli all must be positive. Born and coworkers developed a theory on the stability of crystal lattices [66]-[72]. For tetragonal crystals at ambient conditions, the seven elastic stability criteria are given by

- $2 c_{11}+c_{33}+2 c_{12}+4 c_{13}>0$

- $c_{11}+c_{33}-2 c_{13}>0$

- $c_{12}, c_{33}, c_{44}, c_{66}>0$

- $c_{11}-c_{12}>0$

Note that the number of criteria is reduced for the lower number of elastic constants in hexagonal or cubic crystals to 5 or 3 , respectively. The last condition is used to define the tetragonal shear modulus $c^{\prime}=\left(c_{11}-c_{12}\right) / 2$. In some works, the direct difference $C^{\prime}=c_{11}-c_{12}$ is used. If external, hydrostatic pressure $p$ is applied, then the crystal becomes unstable when $2 p>C^{\prime}$, that is, at $p>c^{\prime}$.

The linear compressibility $\beta$ is the crystal response to hydrostatic pressure by a length decrease. For cubic systems, the linear compressibility is isotropic, that is, a sphere of a cubic crystal under hydrostatic pressure remains a sphere. The situation is different in non-cubic systems where $\beta=\beta(\hat{r})$ becomes directionally dependent. In hexagonal, trigonal, and tetragonal systems, the directional dependence is given by

$$
\beta(\hat{r})=\left(s_{11}+s_{12}+s_{13}\right)\left(\hat{x}^{2}+\hat{y}^{2}\right)-\left(s_{11}+s_{12}-s_{13}-s_{33}\right) \hat{z}^{2} .
$$

The linear compressibility of a cubic crystal is simply $\beta^{\mathrm{cub}}=s_{11}+2 s_{12}$. The volume compressibility $\kappa$ of hexagonal and tetragonal systems is also directionally dependent and given in Reuß's approach by

$$
\kappa(\hat{r})=\left(s_{11}+s_{12}+s_{13}\right)\left(\hat{x}^{2}+\hat{y}^{2}\right)+\left(s_{33}+2 s_{13}\right) \hat{z}^{2}
$$

For cubic systems, $s_{13}=s_{12}$ and $s_{33}=s_{11}$, that is, $\kappa^{\text {cub }}=3\left(s_{11}+2 s_{12}\right)$, and thus, the bulk modulus $B=1 / \kappa$ is isotropic for crystals with cubic symmetry. For hexagonal and tetragonal systems, $\kappa$ becomes isotropic when the two terms $s_{11}+s_{12}+s_{13}$ and $s_{33}+2 s_{13}$ in Equation (21) are equal. Therefore, the anisotropy of the hexagonal and tetragonal bulk moduli is defined by

$$
A_{B}=\frac{s_{33}+2 s_{13}}{s_{11}+s_{12}+s_{13}},
$$

and their isotropic compressibility becomes $\kappa_{\text {iso }}^{\text {hextet }}=2 s_{11}+s_{33}+2 s_{12}+4 s_{13}$.

Other than the bulk modulus of cubic crystals, Young's modulus of cubic, hexagonal, or tetragonal systems is not isotropic. The representation surface of Young's modulus for tetragonal systems with classes $4 \mathrm{~mm}, 422, \overline{4} 2 \mathrm{~m}$, and $4 / \mathrm{mmm}$ is given by 


$$
\frac{1}{E^{t}(\hat{r})}=\left(\hat{x}^{4}+\hat{y}^{4}\right) s_{11}+\hat{z}^{4} s_{33}+\hat{x}^{2} \hat{y}^{2}\left(2 s_{12}+s_{66}\right)+\hat{z}^{2}\left(1-\hat{z}^{2}\right)\left(2 s_{13}+s_{44}\right)
$$

For the tetragonal classes $4,4 / m$, and $\overline{4}$, an additional term is present such that

$$
\frac{1}{E^{t^{\prime}}(\hat{r})}=\frac{1}{E^{t}(\hat{r})}+2 \hat{x} \hat{y}\left(\hat{x}^{2}-\hat{y}^{2}\right) s_{16}
$$

The shear anisotropic factors provide a measure of the degree of anisotropy in the bonding between atoms in different planes. The number of different shear anisotropies depends on the crystal system. In both hexagonal and tetragonal systems, the shear anisotropic factors $A_{100}$ (or equivalent $A_{010}$ ) for the $\{100\}$ shear planes between the $\langle 011\rangle$ and $\langle 010\rangle$ directions and $A_{001}$ for the $\{001\}$ planes between $\langle 110\rangle$ and $\langle 010\rangle$ are:

$$
\begin{aligned}
& A_{100}=\frac{4 c_{44}}{c_{11}+c_{33}-2 c_{13}} \\
& A_{001}=\frac{2 c_{66}}{c_{11}-c_{12}}
\end{aligned}
$$

In cubic crystals, both factors are the same $A_{e}=A_{001}=2 c_{44} /\left(c_{11}-c_{12}\right)$, as mentioned above. In hexagonal systems, $c_{66}=\left(c_{11}-c_{12}\right) / 2$, and thus, $A_{001}=1$. For isotropic crystals, all $A$ factors must be unity, while any value smaller or greater than unity is a measure of the degree of elastic anisotropy possessed by the crystal.

Comparing the Equations (22) and (25) for the elastic anisotropies with the Born-Huang [72] criteria, these equations can clearly be used to show the elastic stability. Most obviously, crystals with one negative anisotropy are not stable. Further, crystals with large anisotropies also tend to instabilities; in particular, crystals are not stable for $A \rightarrow \infty$ when one of the denominators becomes zero. This behavior makes the anisotropies important parameters, even though they may not cover all possible causes for Born-Huang instabilities.

\section{Appendix C. Equations for Calculating Properties from the Elastic Constants}

Besides the elastic moduli, further important physical quantities can be derived from the elastic constants. Acoustical spectroscopy is widely used to determine the elastic properties of crystalline solids. The propagation of sound waves in solids is described by the Christoffel equation:

$$
\left(\Gamma_{i j}-\rho v^{2} \delta_{i j}\right) U_{j}=0,
$$

where $v$ is the phase velocity, $\rho$ is the mass density, $\delta_{i j}$ is the Kronecker delta, $U$ is the polarisation vector, and

$$
\Gamma_{i j}=c_{i j k l} l_{j} l_{l}
$$

is the Christoffel tensor built from the elastic constants and the direction cosines $l_{i} \quad(i=1,2,3)$ that describe the direction of wave motion. For tetragonal 
systems, the Christoffel tensor is given by

$$
\begin{gathered}
\Gamma_{11}=c_{11} l_{1}^{2}+c_{66} l_{2}^{2}+c_{44} l_{3}^{2}+2 c_{16} l_{1} l_{2} \\
\Gamma_{22}=c_{66} l_{1}^{2}+c_{11} l_{2}^{2}+c_{44} l_{3}^{2}-2 c_{16} l_{1} l_{2} \\
\Gamma_{33}=c_{44} l_{1}^{2}+c_{44} l_{2}^{2}+c_{33} l_{3}^{2} \\
\Gamma_{12}=c_{16} l_{1}^{2}-c_{16} l_{2}^{2}+\left(c_{12}+c_{66}\right) l_{1} l_{2}-c_{16} l_{2} l_{3} \\
\Gamma_{13}=\left(c_{13}+c_{44}\right) l_{1} l_{3} \\
\Gamma_{23}=\left(c_{13}+c_{44}\right) l_{2} l_{3}
\end{gathered}
$$

and $\Gamma_{i j}=\Gamma_{j i}$. The Christoffel tensor reduces for the classes $4 \mathrm{~mm}, \overline{4} 2 \mathrm{~m}, 422$, and $4 / \mathrm{mmm}$, where $c_{16}=0$, to

$$
\begin{gathered}
\Gamma_{11}=c_{11} l_{1}^{2}+c_{66} l_{2}^{2}+c_{44} l_{3}^{2} \\
\Gamma_{22}=c_{66} l_{1}^{2}+c_{11} l_{2}^{2}+c_{44} l_{3}^{2} \\
\Gamma_{33}=c_{44} l_{1}^{2}+c_{44} l_{2}^{2}+c_{33} l_{3}^{2} \\
\Gamma_{12}=\left(c_{12}+c_{66}\right) l_{1} l_{2} \\
\Gamma_{13}=\left(c_{13}+c_{44}\right) l_{1} l_{3} \\
\Gamma_{23}=\left(c_{13}+c_{44}\right) l_{2} l_{3}
\end{gathered}
$$

The solution of the characteristic $3 \times 3$ matrix results in a third-order equation in $v^{2}$ for the phase velocity. Three distinct modes appear, one with longitudinal and two with transversal polarisation. Due to possible mixing, these modes are often referred to as quasi-longitudinal or quasi-transversal modes. The longitudinal mode corresponds to a pressure ( $p$-wave) or compression wave as it appears also in gases. On the other hand, the transversal modes appear for solids, and they are distinguished as fast $\left(s_{1}\right)$ and slow ( $s_{2}$-wave) shear waves. The wave properties are presented as slowness surfaces.

The elastic constants also allow direct estimation of the averaged sound velocity $\bar{v}$ from the longitudinal $\left(v_{l}\right)$ and transverse $\left(v_{t}\right)$ elastic wave velocities of isotropic materials, which are given by

$$
\begin{aligned}
& v_{l}=\sqrt{\frac{3 B+4 G}{3 \rho}} \\
& v_{t}=\sqrt{\frac{G}{\rho}}
\end{aligned}
$$

where $\rho$ is the mass density of the material. Here, $\bar{v}$ is approximately predicted by

$$
\bar{v}=\left[\frac{3}{v_{l}^{-3}+2 v_{t}^{-3}}\right]^{1 / 3} .
$$

For low temperatures, where only acoustic vibrational modes contribute, the Debye temperature $\Theta_{D}$ can be estimated from the average sound velocity using the relation [58]: 


$$
\Theta_{D}^{\mathrm{ac}}=\bar{v} \frac{h}{k_{B}} \sqrt[3]{\frac{f}{4 \pi} \frac{N_{A} \rho}{M}}=\bar{v} \frac{h}{k_{B}} \sqrt[3]{\frac{f}{4 \pi} \frac{1}{V_{p}}},
$$

where $h, k_{B}$, and $N_{A}$ are Plank's constant, Boltzman's constant, and Avogadoro's number, respectively. The degree of freedom for $n$ atoms in a primitive cell with volume $V_{p}$ ( $f=12$ for Heusler compounds with $L 2_{1}$ structure) is $f=3 n$, and $M$ is the molecular mass, that is, the sum of all masses of the atoms in the primitive cell of the compound.

In solids, the Grüneisen parameter $\zeta$ is a measure of the anharmonicity of the interactions between the atoms. In general, it is calculated from logarithmic derivatives of the vibrational frequencies with respect to the crystal volume. However, full phonon calculations as function of crystal volumes are demanding tasks, and fast estimates are thus welcome. Belomestnykh [60] derived an "acoustical" Grüneisen parameter $\zeta^{\mathrm{ac}}$ that is directly related to the sound velocities. Therefore, $\zeta^{\mathrm{ac}}$ is given by

$$
\zeta^{\mathrm{ac}}=\frac{3}{2} \frac{\left(3 v_{l}^{2}-4 v_{t}^{2}\right)}{\left(v_{l}^{2}+2 v_{t}^{2}\right)} .
$$

